\title{
Localizations of abelian Eilenberg-Mac Lane spaces of finite type
}

\author{
CARles CASACUBERTA \\ JOSÉ L RODRÍGUEZ \\ JIN-YEN TAI
}

\begin{abstract}
We prove that every homotopical localization of the circle $S^{1}$ is an aspherical space whose fundamental group $A$ is abelian and admits a ring structure with unit such that the evaluation map $\operatorname{End}(A) \rightarrow A$ at the unit is an isomorphism of rings. Since it is known that there is a proper class of nonisomorphic rings with this property, and we show that all occur in this way, it follows that there is a proper class of distinct homotopical localizations of spaces (in spite of the fact that homological localizations form a set). This answers a question asked by Farjoun in the nineties.

More generally, we study localizations $L_{f} K(G, n)$ of Eilenberg-Mac Lane spaces with respect to any map $f$, where $n \geq 1$ and $G$ is any abelian group, and we show that many properties of $G$ are transferred to the homotopy groups of $L_{f} K(G, n)$. Among other results, we show that, if $X$ is a product of abelian Eilenberg-Mac Lane spaces and $f$ is any map, then the homotopy groups $\pi_{m}\left(L_{f} X\right)$ are modules over the ring $\pi_{1}\left(L_{f} S^{1}\right)$ in a canonical way. This explains and generalizes earlier observations made by other authors in the case of homological localizations.
\end{abstract}

55P20, 55P60; 18A40, 16S10

Dedicated to the memory of Professor Rüdiger Göbel

\section{Introduction}

Preliminary versions of this article have circulated for a long time, and parts of it were reported in a survey article by Casacuberta [19]. Since then, some of the ideas contained in it have triggered a considerable amount of work about the preservation of algebraic structures by localizations in homotopy theory and group theory; references are given below. A stable analogue was developed in Casacuberta and Gutiérrez [20] and Gutiérrez [43]. Thus, the presentation of results in the present paper predates a number of sequels that have been published in the meantime. We have kept the core of the paper in its original form, although the present version is more detailed and much 
more self-contained than earlier drafts, partly due to pertinent comments of referees, to whom we are indebted.

One of the initial motivations of the article was the following question posed by Farjoun: for a fixed space $X$, do the distinct homotopy types of the form $L_{f} X$ form a set or instead a proper class if $f$ ranges over all maps? Here $L_{f}$ denotes homotopical localization with respect to a given map $f$ between spaces - the essentials of this theory can be found in Bousfield [8], Farjoun [32] and Hirschhorn [47]. Although it is known that localizations with respect to homology theories form a set from Dwyer and Palmieri [31] and Ohkawa [56], we discovered that there is a proper class of distinct homotopy types of the form $L_{f} S^{1}$, where $S^{1}$ denotes the circle. Similarly, as was later shown in [20], there is a proper class of nonequivalent localizations of the Eilenberg-Mac Lane spectrum $H \mathbb{Z}$ in the homotopy category of spectra.

In Section 1 we give a new proof, based on a study of the interaction of localizations with certain monads, of the fact — first described by Farjoun in [32] — that, for every abelian group $G$, every map $f$ and $n \geq 1$, there are abelian groups $A$ and $B$ such that

$$
L_{f} K(G, n) \simeq K(A, n) \times K(B, n+1) .
$$

Following a personal communication by Bousfield, we prove that $B=0$ when the group $G$ is reduced, ie when it does not contain any nonzero divisible subgroups. In fact, we prove that the group $B$ is determined by a torsion divisible direct summand of $G$. Hence, if $G$ is torsion-free, then $B=0$ as well.

It follows that, for every map $f$, there is an abelian group $A$ such that

$$
L_{f} S^{1} \simeq K(A, 1)
$$

and $A$ admits a unique commutative ring structure with 1 such that the unit morphism $\mathbb{Z} \rightarrow A$ induces an isomorphism of rings $\operatorname{End}(A) \cong A$. (The elements of $\operatorname{End}(A)$ are endomorphisms of $A$ as an abelian group, operating under addition and composition.)

Rings $A$ with this property are called rigid in this article; elsewhere, they are called $E$-rings, a name first used by Schultz in [70]. Correspondingly, rings $A$ such that the multiplication map $A \otimes A \rightarrow A$ is bijective were called $T$-rings in Bowshell and Schultz [12], although $T$-rings are normally called solid rings by topologists; see Bousfield [5] and Bousfield and Kan [11].

Besides the fact that solid rings are rigid (as shown in Theorem 3.6 below or already in [12] with a different naming), we have chosen this terminology in order to emphasize that rigid rings have as few additive endomorphisms as possible. A further justification comes from the fact that a ring is rigid if and only if its underlying abelian group admits only one multiplication with a fixed left identity element; see Theorem 3.3. 
The rigid rings turn out to be precisely the localizations of $\mathbb{Z}$ in the category of groups, while the solid rings are the $\mathbb{Z}$-epimorphs, ie rings for which the unit morphism $\mathbb{Z} \rightarrow A$ is an epimorphism of rings. The $p$-adics $\widehat{\mathbb{Z}}_{p}$ and products $\mathbb{Z}[1 / p] \times \mathbb{Z}[1 / q]$ with $p \neq q$ are examples of rigid rings which are not solid. Torsion-free rigid rings of finite rank are well understood; see Pierce and Vinsonhaler [60].

More generally, if $R$ is any commutative ring, we say that an $R$-algebra $A$ with 1 is rigid if evaluation at 1 yields an isomorphism of $R$-algebras $\operatorname{End}_{R}(A) \cong A$. Some basic properties of rigid $R$-algebras are described in Section 3, where we generalize earlier results of Bowshell and Schultz $[12 ; 70]$. An updated presentation of results about rigid $R$-algebras can be found in Göbel and Trlifaj [41, Chapter 13], where they are named $E(R)$-algebras. See also Göbel and Goldsmith [36] and Göbel and Trlifaj [42].

Neither the field of reals $\mathbb{R}$ nor the $p$-adic field $\widehat{\mathbb{Q}}_{p}$ for any prime $p$ admit a rigid ring structure. Thus, there is no analogue of a rationalization functor where $\mathbb{Q}$ is replaced by $\mathbb{R}$, nor a completion functor taking values in $\widehat{\mathbb{Q}}_{p}$.

It is known that there exist rigid rings of arbitrarily large cardinality; see Dugas, Mader and Vinsonhaler [30], and also [42]. Since every rigid ring $A$ occurs as the fundamental group of $L_{f} S^{1}$ for a certain map $f$ (namely, the map $f: S^{1} \rightarrow K(A, 1)$ induced by the inclusion of 1 into $A$ ), we infer that there is a proper class of distinct homotopy types of the form $L_{f} S^{1}$, where $f$ ranges over all possible maps. This answers Farjoun's question referred to above.

The knowledge of the ring $A=\pi_{1}\left(L_{f} S^{1}\right)$ gives important information about the effect of the functor $L_{f}$ on other spaces. Specifically, as we show in Theorem 7.1, the homotopy groups of the $f$-localization of any GEM (that is, any product of abelian Eilenberg-Mac Lane spaces) are then $A$-modules. If $A$ is finite, then the $f$-localization of any GEM is a $K(G, 1)$. In this context, a problem remains unsolved at the time of publication of this article, in spite of the efforts made by several people: to prove or disprove that if $\pi_{1}\left(L_{f} S^{1}\right)$ is finite then every $f$-local space is a $K(G, 1)$.

On the other hand, if $A$ is not cyclic, then there is a set of primes $P$ such that the higher homotopy groups of the $f$-localization of any GEM are either $P$-local (when $A / \mathbb{Z}$ is torsion) or Ext- $P$-complete otherwise. To prove this, we rely on [8, Lemma 5.5]; the set $P$ consists of those primes $p$ such that multiplication by $p$ is an automorphism of $A / \mathbb{Z}$. This result sheds additional light on earlier calculations by Bousfield [7] and Mislin [54] of homological localizations of Eilenberg-Mac Lane spaces.

Possibly the main conceptual finding of this article, which has proved to be fruitful in subsequent work, is that many kinds of algebraic structures are preserved by localizations. This came from observing that, if $G$ is any abelian group (not necessarily finitely 
generated), then the group $A=\pi_{n}\left(L_{f} K(G, n)\right)$ can be described as a localization of $G$ in the category of groups with respect to a certain homomorphism; indeed, $A \cong L_{\alpha} G$, where $\alpha$ is the homomorphism $G \rightarrow A$ induced by the localization map $K(G, n) \rightarrow L_{f} K(G, n)$. In this article, we use this fact to show that $A$ inherits many properties of $G$, and it does so in a unique way. For instance, if $R$ is a commutative ring, then the $n^{\text {th }}$ homotopy group of every localization of a $K(R, n)$ admits a unique compatible structure of a rigid $R$-algebra. Some of our results about rigid algebras were used and extended in Strüngmann's thesis [72], as well as in Braun and Göbel [13] and Göbel and Strüngmann [40], and also [36].

Classes of groups that are closed under localizations (or fail to be closed) were studied in Badzioch and Feshbach [3], Libman [49], O'Sullivan [57], Rodríguez, Scherer and Thévenaz [66], and Rodríguez, Scherer and Viruel [67; 68]. Special cases were worked out by Rodríguez, Scherer and Strüngmann [65] for torsion abelian groups and by Dugas [27; 28] for torsion-free abelian groups. In Göbel, Rodríguez and Shelah [37], and in Göbel and Shelah [39], it was proved that every nonabelian finite simple group admits a proper class of nonisomorphic localizations. Other results about localizations of finite simple groups were obtained by Parker and Saxl [58].

Dual results relating cellularization of spaces with colocalization of groups or modules were first obtained by Rodríguez and Scherer in [63; 64]. The study of cellular covers of groups and modules, as well as the preservation of algebraic structures by such functors, has been an active subject of research; see Blomgren, Chachólski, Farjoun and Segev [4], Buckner and Dugas [15; 16], Chachólski, Damian, Farjoun and Segev [24], Dugas [29], Farjoun, Göbel and Segev [33], Farjoun, Göbel, Segev and Shelah [34], Fuchs and Göbel [35], Göbel, Rodríguez and Strüngmann [38], and Rodríguez and Strüngmann [69], or the thorough survey contained in [42, Part VI].

Cellularizations of stable structures were discussed by Gutiérrez [45]. In [20] it was shown that, for similar reasons as in this article, exact $f$-localizations in the stable homotopy category convert ring spectra (in the homotopical sense) into ring spectra, and module spectra into module spectra, although connectivity assumptions are required in the case of nonexact localizations, ie those not commuting with suspension. The same facts are actually true for strict rings and strict modules in monoidal model categories of spectra, as shown by Casacuberta, Gutiérrez, Moerdijk and Vogt in [21]. This stronger phenomenon is closely related to the preservation of loop spaces by localizations - first proved in $[8 ; 32]$ - and is explained by the interaction of homotopical localizations with certain operads [21].

Acknowledgements This study originated from conversations between the authors and Wojciech Chachólski. Our insight on rigid rings owes much to Warren Dicks, 
Alberto Facchini, and Rüdiger Göbel, whose advice and interest we appreciate. We thank especially Emmanuel Dror Farjoun for many helpful indications.

The article was begun during a stay of the authors at The Fields Institute in Toronto. Casacuberta and Rodríguez were partially supported by Generalitat de Catalunya under grants 1995BEAI400083, 1996BEAI200187, 2009 SGR 119, 2014 SGR 114, and by the Spanish Ministry of Science through the projects PB97-0202, BFM2001-2031, MTM2004-03629, MTM2007-63277, MTM2010-15831, and MTM2013-42178-P. Rodríguez was supported by Junta de Andalucía grant FQM-213.

\section{Localizations of Eilenberg-Mac Lane spaces}

All spaces in this article are meant to be pointed CW-complexes, except for auxiliary occurrences of mapping spaces. Maps preserve base points, and $[X, Y]$ denotes the set of pointed homotopy classes of maps $X \rightarrow Y$.

Let $f: W \rightarrow V$ be any map. A space $X$ is called $f$-local if the induced map of unpointed mapping spaces

$$
\operatorname{map}(f, X): \operatorname{map}(V, X) \longrightarrow \operatorname{map}(W, X)
$$

is a weak homotopy equivalence. If $X$ is connected, then it is $f$-local if and only if the induced map of pointed mapping spaces

$$
\operatorname{map}_{*}(f, X): \operatorname{map}_{*}(V, X) \longrightarrow \operatorname{map}_{*}(W, X)
$$

is a weak homotopy equivalence. As observed in [8, Lemma 2.1], this follows by applying the functor $\operatorname{map}_{*}(-, X)$ to the cofiber sequences $S^{0} \rightarrow W_{+} \rightarrow W$ and $S^{0} \rightarrow V_{+} \rightarrow V$, where the subscript + denotes a disjoint base point.

A map $g: Y \rightarrow Z$ is called an $f$-equivalence if

$$
\operatorname{map}(g, X): \operatorname{map}(Z, X) \longrightarrow \operatorname{map}(Y, X)
$$

is a weak homotopy equivalence for each $f$-local space $X$. It follows from the definition that, for every $f$, the class of $f$-local spaces is closed under homotopy limits (in particular, $\Omega X$ is $f$-local whenever $X$ is $f$-local), and the class of $f$-equivalences is closed under homotopy colimits.

An $f$-localization of a space $X$ is a map $\eta_{X}: X \rightarrow L_{f} X$ which is an $f$-equivalence and where $L_{f} X$ is $f$-local. Such a map exists for all $X$ and for every choice of $f$, and it is unique up to homotopy. Proofs are given, using different techniques, in $[6 ; 32$; 47]. The map $\eta_{X}$ is initial in the pointed homotopy category among maps from $X$ into 
$f$-local spaces, and terminal among $f$-equivalences going out of $X$. Thus $\left(L_{f}, \eta\right)$ is an idempotent monad on the pointed homotopy category. The natural transformation $L_{f} L_{f} \rightarrow L_{f}$ is an isomorphism, and hence it is omitted from the notation. From general properties of idempotent monads [19], it follows that, among other things, a map $g: Y \rightarrow Z$ is an $f$-equivalence if and only if $L_{f}(g): L_{f} Y \rightarrow L_{f} Z$ is a homotopy equivalence, and if and only if it induces a bijection $[Z, X] \cong[Y, X]$ for every $f$-local space $X$. Similarly, a space $X$ is $f$-local if and only if every $f$-equivalence $g: Y \rightarrow Z$ induces a bijection $[Z, X] \cong[Y, X]$. It also follows that every homotopy retract of an $f$-local space is $f$-local, and every homotopy retract of an $f$-equivalence is an $f$-equivalence.

As explained by Tai in [73], $L_{f}$ sends connected spaces to connected spaces. Moreover, if the induced map $\pi_{0}(f)$ of connected components is not bijective, then $L_{f} X$ is contractible for all nonempty spaces $X$.

If the map $f$ is of the form $W \rightarrow *$ (where $*$ denotes a one-point space and $W$ is connected), then $f$-local spaces are called $W$-null. Thus, $Y$ is $W$-null if and only if the pointed mapping $\operatorname{space}^{\operatorname{map}_{*}}(W, Y)$ is weakly contractible. For $f: W \rightarrow *$, it is customary to use the notation $P_{W}$ instead of $L_{f}$, and call it $W$-nullification (the choice of the letter $P$ was due to the fact that Postnikov sections are special cases).

From now on, $K(G, n)$ will denote an Eilenberg-Mac Lane space where $G$ is assumed abelian if $n=1$. The departure point of this article is the fact that, if $f$ is any map, then

$$
L_{f} K(G, n) \simeq K(A, n) \times K(B, n+1)
$$

for some abelian groups $A$ and $B$. This was shown by Farjoun in [32, Section 4.B]. In Theorem 1.3 below, we give an alternative proof of (1-1) using general properties of algebras over monads, and we strengthen the result by showing that to each map $f$ one can functorially associate a homomorphism $g$ of commutative topological monoids such that $L_{f} K(G, n) \simeq L_{g} K(G, n)$ for every $K(G, n)$. We are indebted to an anonymous referee for indicating this fact. It is interesting to note the analogy with [44, Proposition 3.2] in the stable homotopy category.

The infinite symmetric product $\mathrm{SP}^{\infty} X$ of a pointed CW-complex $X$ is the colimit of the quotients $\mathrm{SP}^{k} X=X^{k} / \Sigma_{k}$, where $X^{k}$ denotes the product of $k$ copies of $X$ (with the compactly generated topology) and $\Sigma_{k}$ is the symmetric group on $k$ elements acting by permutations of the factors. The inclusion $\mathrm{SP}^{k} X \subset \mathrm{SP}^{k+1} X$ is given by placing the base point in the additional component.

The space $\mathrm{SP}^{\infty} X$ is a commutative topological monoid (operating by juxtaposition), and it is free as such on $X$. Therefore, $\mathrm{SP}^{\infty}$ may be viewed as the composite of a free-forgetful adjoint pair between the category of pointed spaces and the category of 
commutative topological monoids with the unit element as base point. In fact, there are natural transformations $\iota: \mathrm{Id} \rightarrow \mathrm{SP}^{\infty}$ (corresponding to the inclusion of $X=\mathrm{SP}^{1} X$ into $\mathrm{SP}^{\infty} X$ for each $X$ ) and $\mu: \mathrm{SP}^{\infty} \mathrm{SP}^{\infty} \rightarrow \mathrm{SP}^{\infty}$ defining a monad on spaces.

Recall that, if $(T, \iota, \mu)$ is a monad on any category [50], a T-algebra structure on an object $X$ is a morphism $\rho: T X \rightarrow X$ such that $\rho \circ \iota_{X}=\operatorname{id}_{X}$ and $\rho \circ \mu_{X}=\rho \circ T \rho$. As pointed out in [52, Section 3], the algebras over $\mathrm{SP}^{\infty}$ are the algebras over the commutative operad, namely commutative topological monoids. Thus the free-forgetful adjunction is the Eilenberg-Moore factorization of $\mathrm{SP}^{\infty}$ as a monad on spaces.

Since the functor $\mathrm{SP}^{\infty}$ preserves homotopy equivalences, the monad $\left(\mathrm{SP}^{\infty}, \iota, \mu\right)$ descends to the homotopy category. In what follows, we shall be interested in the Eilenberg-Moore factorization of $\mathrm{SP}^{\infty}$ as a monad on the pointed homotopy category. Let us denote by $[-,-]_{\mathrm{SP}^{\infty}}$ the corresponding morphism set. The adjunction yields bijections

$$
\left[\mathrm{SP}^{\infty} X, Y\right]_{\mathrm{SP}} \cong[X, U Y]
$$

for every space $X$ and every $\operatorname{SP}^{\infty}$-algebra $Y$, where $U$ is the forgetful functor.

A generalized Eilenberg-Mac Lane space (in short, a GEM) is a pointed connected space $X$ with $\pi_{1}(X)$ abelian and such that $X \simeq \prod_{n=1}^{\infty} K\left(\pi_{n}(X), n\right)$. Here and below, we mean the weak product (ie the direct limit of products of a finite number of factors), although this does not change the homotopy type.

As shown by Dold and Thom in [26], $\pi_{n}\left(\operatorname{SP}^{\infty} X\right) \cong H_{n}(X)$ for $n \geq 1$. Therefore, if $M(G, n)$ is a Moore space with $G$ abelian and $H_{n}(M(G, n)) \cong G$, then $\mathrm{SP}^{\infty} M(G, n)=K(G, n)$. This implies the following (and so, in particular, $\mathrm{SP}^{\infty} X$ is a GEM for all connected spaces $X$ ).

Proposition 1.1 A pointed connected space $X$ is a GEM if and only if it is the underlying space of an $\mathrm{SP}^{\infty}$-algebra in the pointed homotopy category, which is then unique up to isomorphism.

Proof Let $\rho: \mathrm{SP}^{\infty} X \rightarrow X$ be an $\mathrm{SP}^{\infty}$-algebra structure on a space $X$. Then $X$ is a retract of $\mathrm{SP}^{\infty} X$, and hence $\pi_{1}(X)$ is abelian. For each $n \geq 1$, choose a map $\alpha_{n}: M\left(\pi_{n}(X), n\right) \rightarrow X$ inducing an isomorphism on $\pi_{n}$. Together, these yield a map

$$
\alpha: \bigvee_{n=1}^{\infty} M\left(\pi_{n}(X), n\right) \longrightarrow X,
$$

corresponding by (1-2) to an $\mathrm{SP}^{\infty}$-algebra map $\beta$ : $\prod_{n=1}^{\infty} K\left(\pi_{n}(X), n\right) \rightarrow X$, namely $\beta=\rho \circ \mathrm{SP}^{\infty} \alpha$, whose underlying map of spaces is a homotopy equivalence. Moreover, 
$\beta$ is an isomorphism of $\mathrm{SP}^{\infty}$-algebras, since any homotopy inverse of $\beta$ is also an $\mathrm{SP}^{\infty}$-algebra map. This proves that the space $X$ is a GEM (compare with [32, Lemma 4.B.2.1], [46, Corollary 4.K.7], or [51, Theorem 24.5]), and it also proves that the $\mathrm{SP}^{\infty}$-algebra structure on $X$ is unique up to isomorphism.

Conversely, every GEM $X$ is homotopy equivalent to $\prod_{n=1}^{\infty} K\left(\pi_{n}(X), n\right)$, which admits a (componentwise) commutative monoid structure, hence an $\mathrm{SP}^{\infty}$-algebra structure in the category of spaces, which passes to the homotopy category.

For an idempotent monad $(L, \eta)$ on a category, the morphisms $\varphi$ such that $L \varphi$ is an isomorphism are called $L$-equivalences and the objects isomorphic to $L X$ for some $X$ are called $L$-local. A functor $T$ is said to preserve $L$-equivalences if $T \varphi$ is an $L$-equivalence whenever $\varphi$ is an $L$-equivalence.

Theorem 1.2 Let $(T, \iota, \mu)$ be a monad on a category and let $(L, \eta)$ be an idempotent monad on the same category. If the functor $T$ preserves $L$-equivalences, then for every $T$-algebra structure $\rho: T X \rightarrow X$ on an object $X$ there is a unique $T$-algebra structure on $L X$ such that $\eta_{X}: X \rightarrow L X$ is a morphism of $T$-algebras.

Proof Since $\eta_{X}: X \rightarrow L X$ is an $L$-equivalence, $T \eta_{X}$ is an $L$-equivalence by assumption. Therefore, there is a unique morphism $\sigma: T L X \rightarrow L X$ such that

$$
\sigma \circ T \eta_{X}=\eta_{X} \circ \rho .
$$

As we next check, $\sigma$ is a $T$-algebra structure on $L X$, and (1-3) says then that $\eta_{X}$ is a morphism of $T$-algebras. The fact that $\sigma \circ \iota_{L X}=\mathrm{id}_{L X}$ follows from the equalities

$$
\sigma \circ \iota_{L X} \circ \eta_{X}=\sigma \circ T \eta_{X} \circ \iota_{X}=\eta_{X} \circ \rho \circ \iota_{X}=\eta_{X}
$$

and from the fact that two morphisms $L X \rightarrow L X$ are equal if and only if their composites with $\eta_{X}: X \rightarrow L X$ are equal. Similarly, one proves that $\sigma \circ \mu_{L X}=\sigma \circ T \sigma$ by checking that the composites of both members of this expression with $T T \eta_{X}$ are equal:

$$
\begin{aligned}
\sigma \circ \mu_{L X} \circ T T \eta_{X} & =\sigma \circ T \eta_{X} \circ \mu_{X}=\eta_{X} \circ \rho \circ \mu_{X} \\
& =\eta_{X} \circ \rho \circ T \rho=\sigma \circ T \eta_{X} \circ T \rho=\sigma \circ T \sigma \circ T T \eta_{X},
\end{aligned}
$$

and then using the fact that $T T \eta_{X}$ is an $L$-equivalence, since $T$ preserves $L$-equivalences by assumption.

Now we can prove the following result, in which part 4 is new, while the rest is essentially contained in [32, Section 4.B]. The preservation of GEMs by localizations was also discussed by Badzioch in [2] and by Bousfield in [9, Corollary 2.11]. 
Theorem 1.3 The following claims are true for every map $f$ of pointed spaces:

(1) $\mathrm{SP}^{\infty}$ preserves $f$-equivalences.

(2) $L_{f}$ sends GEMs to GEMs.

(3) For every abelian group $G$ and $n \geq 1$, there are abelian groups $A$ and $B$ such that $L_{f} K(G, n) \simeq K(A, n) \times K(B, n+1)$.

(4) If $X$ is a GEM, then $L_{f} X \simeq L_{\mathrm{SP}^{\infty}} X$.

Proof As shown in [32, Section 1.G], the functor $L_{f}$ commutes with finite products up to homotopy. Hence, if $g: X \rightarrow Y$ is any $f$-equivalence, then the $k$-fold product $g^{k}$ is an $f$-equivalence for all $k$. In order to show that $\mathrm{SP}^{k} g$ is also an $f$-equivalence for all $k$, one may argue as in [32, Section 4.A] as follows. For each space $X$, the symmetric product $\mathrm{SP}^{k} X=X^{k} / \Sigma_{k}$ is a colimit of the diagram from $\Sigma_{k}$ (viewed as a category with one object) to the category of spaces sending the single object to $X^{k}$ and each element of $\Sigma_{k}$ to the corresponding automorphism of $X^{k}$. This diagram is not free. However, $\mathrm{SP}^{k} X$ is also the colimit of the diagram indexed by the opposite of the category of orbits of $\Sigma^{k}$ sending each quotient $\Sigma_{k} / H$ to the fixedpoint subspace $\left(X^{k}\right)^{H}$ and each translation self-map of $\Sigma_{k} / H$ to the corresponding automorphism. Now this diagram is free, and therefore $\mathrm{SP}^{k} X$ is its homotopy colimit. Note that this diagram takes values in fixed-point subspaces of actions of subgroups of $\Sigma_{k}$ on $X^{k}$, and each such subspace is homeomorphic to $X^{n}$ for some $n \leq k$ (compare with [2, Remark 3.1]). This shows that the map $\mathrm{SP}^{k} g$ is a homotopy colimit of a diagram taking values in $g^{n}$ with $n \leq k$. Since the class of $f$-equivalences is closed under homotopy colimits, we may infer that $\mathrm{SP}^{k} g$ is an $f$-equivalence for all $k$. Finally, note that $\mathrm{SP}^{\infty} g$ is not only the colimit of the sequence $\mathrm{SP}^{k} g$, but it is, in fact, a homotopy colimit, since all the arrows in the sequence are inclusions. Therefore, $\mathrm{SP}^{\infty} g$ is an $f$-equivalence.

In order to prove (2) and (3), we may use Theorem 1.2 to endow $L_{f} K(G, n)$ with an $\mathrm{SP}^{\infty}$-algebra structure in the pointed homotopy category such that the localization map $\eta: K(G, n) \rightarrow L_{f} K(G, n)$ is an algebra map. By Proposition 1.1, $L_{f} K(G, n)$ is a GEM, and hence is isomorphic (as an $\mathrm{SP}^{\infty}$-algebra) to $\prod_{i=1}^{\infty} K\left(A_{i}, i\right)$ where $A_{i}=\pi_{i}\left(L_{f} K(G, n)\right)$. As a special case of (1-2), we have a bijection

$$
\left[K(G, n), L_{f} K(G, n)\right]_{\mathrm{SP}} \infty \cong\left[M(G, n), L_{f} K(G, n)\right],
$$

where the algebra structure of $L_{f} K(G, n)$ is neglected in the right-hand term. The set $\left[M(G, n), L_{f} K(G, n)\right]$ is the product of the sets $\left[M(G, n), K\left(A_{i}, i\right)\right]$, which are possibly nonzero only for

$H^{n}\left(M(G, n) ; A_{n}\right) \cong \operatorname{Hom}\left(G, A_{n}\right) \quad$ and $\quad H^{n+1}\left(M(G, n) ; A_{n+1}\right) \cong \operatorname{Ext}\left(G, A_{n+1}\right)$. 
Therefore, the map $M(G, n) \rightarrow L_{f} K(G, n)$ corresponding to the localization map $\eta$ under (1-4) has $M(G, n) \rightarrow K\left(A_{n}, n\right) \times K\left(A_{n+1}, n+1\right)$ as a homotopy retract. From the fact that the projections $L_{f} K(G, n) \rightarrow K\left(A_{i}, i\right)$ are $\mathrm{SP}^{\infty}$-algebra maps, it then follows that $\eta$ has a retract

$$
\xi: K(G, n) \longrightarrow K\left(A_{n}, n\right) \times K\left(A_{n+1}, n+1\right) .
$$

Forgetting the $\mathrm{SP}^{\infty}$-algebra structure, we conclude that $\xi$ is an $f$-localization, since every homotopy retract of an $f$-equivalence is an $f$-equivalence, and every homotopy retract of an $f$-local space is $f$-local. This proves (3).

In order to prove (4), since both $L_{f}$ and $L_{\mathrm{SP}} \infty_{f}$ preserve GEMs, it is sufficient to check that a GEM is $f$-local if and only if it is $\operatorname{SP}^{\infty} f$-local. Note first that, by (1), $\mathrm{SP}^{\infty} f$ is an $f$-equivalence, and this implies that every $f$-local space is $\mathrm{SP}^{\infty} f$-local. For the converse, let $X$ be a GEM and choose a structure map $\rho: \mathrm{SP}^{\infty} X \rightarrow X$. Suppose that $X$ is $\mathrm{SP}^{\infty} f$-local, that is, $\operatorname{map}\left(\operatorname{SP}^{\infty} f, X\right)$ is a weak equivalence. Observe that the composite

$$
\operatorname{map}(f, X) \longrightarrow \operatorname{map}\left(\mathrm{SP}^{\infty} f, \mathrm{SP}^{\infty} X\right) \longrightarrow \operatorname{map}\left(\mathrm{SP}^{\infty} f, X\right),
$$

where the second arrow is induced by $\rho$, is a homotopy right inverse of the map induced by the unit $\iota_{f}: f \rightarrow \operatorname{SP}^{\infty} f$. Hence $\operatorname{map}(f, X)$, as a homotopy retract of $\operatorname{map}\left(\operatorname{SP}^{\infty} f, X\right)$, is a weak equivalence as well; so $X$ is $f$-local. This concludes the proof.

One consequence of Theorem 1.3 is that nullifications of GEMs can be explicitly described in terms of classical localizations and completions at primes, since nullifications with respect to Moore spaces are well understood [10, Theorem 7.5].

Corollary 1.4 If $W$ is any connected space and $X$ is a GEM, then there is a wedge $M=\bigvee_{i=1}^{\infty} M\left(G_{i}, i\right)$ of Moore spaces such that $P_{W} X \simeq P_{M} X$.

Proof Write $\mathrm{SP}^{\infty} W \simeq \prod_{i=1}^{\infty} K\left(G_{i}, i\right)$ for a family of abelian groups $G_{i}$, and choose $M=\bigvee_{i=1}^{\infty} M\left(G_{i}, i\right)$. Then $\mathrm{SP}^{\infty} M \simeq \mathrm{SP}^{\infty} W$, and hence, by part 4 of Theorem 1.3,

$$
P_{W} X \simeq P_{\mathrm{SP}^{\infty}} X \simeq P_{\mathrm{SP}^{\infty}} X \simeq P_{M} X .
$$

Hence, for example, there are very few homotopy types of the form $P_{W} S^{1}$, where $W$ is any space (in fact, either $P_{W} S^{1} \simeq S^{1}$ or $P_{W} S^{1} \simeq *$; see Corollary 5.10). However, as we show next, $f$-localizations of GEMs are more involved if $f: W \rightarrow V$ is a map where neither $W$ nor $V$ are contractible. Among other features, there is a proper class of distinct homotopy types of the form $L_{f} S^{1}$. 
Part 1 of Theorem 1.3, stating that $\mathrm{SP}^{\infty}$ preserves $f$-equivalences for every $f$, can be generalized as follows. If $E$ is any (homotopy) ring spectrum, then $X \mapsto E \wedge X$ defines a monad on the homotopy category of spectra, and $X \mapsto \Omega^{\infty}\left(E \wedge \Sigma^{\infty} X\right)$ defines a monad on the pointed homotopy category of spaces, where the functor $\Sigma^{\infty}$ sends each space to its suspension spectrum and $\Omega^{\infty}$ is its left adjoint. If $E$ is chosen to be the Eilenberg-Mac Lane spectrum $H \mathbb{Z}$, then each connected component of $\Omega^{\infty}\left(H \mathbb{Z} \wedge \Sigma^{\infty} X\right)$ is a GEM for all $X$ (see [9, Section 2] or [20, Proposition 5.3]), and the natural map

$$
\mathrm{SP}^{\infty} X \longrightarrow \Omega^{\infty}\left(H \mathbb{Z} \wedge \Sigma^{\infty} X\right)
$$

induces isomorphisms of $\pi_{n}$ for $n \geq 1$.

Theorem 1.5 Let $f$ be any map and $R$ a ring with 1 . Then the following hold:

(a) The monad $X \mapsto \Omega^{\infty}\left(H R \wedge \Sigma^{\infty} X\right)$ preserves $f$-equivalences.

(b) If $X$ is a GEM and each of the homotopy groups of $X$ is equipped with a left $R$-module structure, then the homotopy groups of $L_{f} X$ also admit left $R$-module structures.

Proof The functor $\Sigma^{\infty}$ sends $f$-equivalences of spaces to $\Sigma^{\infty} f$-equivalences of spectra, while smashing with $H R$ preserves $\Sigma^{\infty} f$-equivalences (see [9;20]), and $\Omega^{\infty}$ sends $\Sigma^{\infty} f$-equivalences to $f$-equivalences, since $L_{f} \Omega^{\infty} E \simeq \Omega^{\infty} L_{\Sigma} f_{f} E$ for every spectrum $E$ as shown in [9, Theorem 2.10] for nullifications and in [22] in full generality. This proves (a).

In order to prove (b), recall from [20, Section 5] that the connected algebras over the monad $X \mapsto \Omega^{\infty}\left(H R \wedge \Sigma^{\infty} X\right)$ are precisely the GEMs equipped with a left $R$-module structure on each of their homotopy groups. Since this monad preserves $f$-equivalences by part (a), Theorem 1.2 implies our claim.

\section{Algebraic formulas}

From part 3 of Theorem 1.3, in this section we derive algebraic formulas involving the homotopy groups of an arbitrary localization of a $K(G, n)$. For an abelian group $G$, an integer $n \geq 1$ and a map $f$, let

$$
\eta: K(G, n) \longrightarrow K(A, n) \times K(B, n+1)
$$

be the $f$-localization map. Then $\eta$ is determined, up to homotopy, by its composites with the projections onto the two factors, which correspond to elements $\alpha \in \operatorname{Hom}(G, A)$ and $\beta \in \operatorname{Ext}(G, B)$. Not all elements of $\operatorname{Hom}(G, A)$ and $\operatorname{Ext}(G, B)$ can occur, but the 
following conditions are necessary and sufficient. For each element $\beta \in \operatorname{Ext}(G, B)$, we choose a representing exact sequence

$$
0 \longrightarrow B \longrightarrow E \longrightarrow G \longrightarrow 0
$$

and consider the connecting homomorphism $\delta: \operatorname{Hom}(B, B) \rightarrow \operatorname{Ext}(G, B)$.

Theorem 2.1 Let $G$ be an abelian group and let $n \geq 2$. Let $A$ and $B$ be abelian groups and suppose given elements $\alpha \in \operatorname{Hom}(G, A)$ and $\beta \in \operatorname{Ext}(G, B)$. Then there exists a map $f$ such that the $f$-localization $L_{f} K(G, n)$ is determined by $\alpha$ and $\beta$ if and only if $\alpha$ and the connecting homomorphism $\delta$ associated with $\beta$ induce isomorphisms

(1) $\operatorname{Hom}(A, A) \cong \operatorname{Hom}(G, A)$,

(2) $\operatorname{Hom}(A, B) \cong \operatorname{Hom}(G, B)$,

(3) $\operatorname{Ext}(A, B) \oplus \operatorname{Hom}(B, B) \cong \operatorname{Ext}(G, B)$.

If $n=1$, then the same claim is true if we add the condition that $\alpha$ induces

(4) $\operatorname{Hom}\left(H_{2}(A), B\right) \cong \operatorname{Hom}\left(H_{2}(G), B\right)$.

Proof Suppose first that $L_{f} K(G, n) \simeq K(A, n) \times K(B, n+1)$ for a map $f$, and suppose that $\eta: K(G, n) \rightarrow L_{f} K(G, n)$ is determined by $\alpha$ and $\beta$. Then, since the class of $f$-local spaces is closed under loops and retracts, each of $K(A, n), K(B, n+1)$ and $K(B, n)$ is $f$-local. Since $\eta$ is an $f$-equivalence, the fact that $K(A, n)$ is $f$-local tells us that $\eta$ induces an isomorphism

$$
H^{n}(K(A, n) \times K(B, n+1) ; A) \cong H^{n}(K(G, n) ; A),
$$

which yields the isomorphism (1). Similarly, the fact that $K(B, n)$ is $f$-local implies that $\alpha$ induces (2). The fact that $K(B, n+1)$ is $f$-local yields

$$
H^{n+1}(K(A, n) \times K(B, n+1) ; B) \cong H^{n+1}(K(G, n) ; B) .
$$

Here we need to recall from [75, Theorem V.7.8] that $H_{n+1}(K(G, n))=0$ for every abelian group $G$ if $n \geq 2$. Hence, if $n \geq 2$, then (2-2) is equivalent to (3), where the isomorphism is induced by $\alpha$ on the first summand and is equal to $\delta$ on the second summand. If $n=1$, then (2-2) is equivalent to (3) together with (4).

Conversely, assume that conditions (1)-(3) hold, and that condition (4) also holds if $n=1$. Let $X=K(A, n) \times K(B, n+1)$, and let $f: K(G, n) \rightarrow X$ be given by $\alpha$ and $\beta$. Then $\operatorname{map}_{*}(K(G, n), X)$ is a group-like space whose only possibly nonzero homotopy groups are $\operatorname{Hom}(G, A) \oplus \operatorname{Ext}(G, B)$ in dimension 0 and $\operatorname{Hom}(G, B)$ in dimension 1 , while if $n \geq 2$ then $\operatorname{map}_{*}(X, X)$ has

$$
\operatorname{Hom}(A, A) \oplus \operatorname{Ext}(A, B) \oplus \operatorname{Hom}(B, B)
$$


in dimension 0 and $\operatorname{Hom}(A, B)$ in dimension 1 . Thus, the given conditions ensure that $X$ is $f$-local. Since $f$ is indeed an $f$-equivalence, it follows that $X \simeq L_{f} K(G, n)$. The case $n=1$ is treated analogously using (4).

Lemma 2.2 Let $\alpha \in \operatorname{Hom}(G, A)$ and $\beta \in \operatorname{Ext}(G, B)$ be given, where $G, A$ and $B$ are abelian groups. Let $N$ be the kernel of $\alpha$, and let $\delta: \operatorname{Hom}(B, B) \rightarrow \operatorname{Ext}(G, B)$ be the connecting homomorphism associated with $\beta$. If $\alpha$ and $\delta$ induce isomorphisms

(1) $\operatorname{Hom}(A, B) \cong \operatorname{Hom}(G, B)$ and

(2) $\operatorname{Ext}(A, B) \oplus \operatorname{Hom}(B, B) \cong \operatorname{Ext}(G, B)$,

then

$\left(1^{\prime}\right) \operatorname{Hom}(N, B)=0$, and

$\left(2^{\prime}\right) i^{*} \circ \delta: \operatorname{Hom}(B, B) \rightarrow \operatorname{Ext}(N, B)$ is an isomorphism, where $i: N \rightarrow G$ denotes the inclusion.

Furthermore, $\left(1^{\prime}\right)$ and $\left(2^{\prime}\right)$ imply (1) and (2) if $\alpha$ is surjective.

Proof Consider the exact sequence

$$
0 \longrightarrow N \stackrel{i}{\longrightarrow} G \stackrel{\alpha}{\longrightarrow} Q \longrightarrow 0,
$$

where $Q=\operatorname{Im} \alpha$, and the associated long exact sequence

$$
0 \longrightarrow \operatorname{Hom}(Q, B) \longrightarrow \operatorname{Hom}(G, B) \longrightarrow \operatorname{Hom}(N, B)
$$$$
\longrightarrow \operatorname{Ext}(Q, B) \longrightarrow \operatorname{Ext}(G, B) \longrightarrow \operatorname{Ext}(N, B) \longrightarrow 0 .
$$

Consider also the factorization

$$
\operatorname{Hom}(A, B) \longrightarrow \operatorname{Hom}(Q, B) \longrightarrow \operatorname{Hom}(G, B)
$$

of $\alpha^{*}$, where the second arrow is injective, and, similarly,

$$
\operatorname{Ext}(A, B) \longrightarrow \operatorname{Ext}(Q, B) \longrightarrow \operatorname{Ext}(G, B),
$$

where the first arrow is surjective. From the isomorphism $\operatorname{Hom}(A, B) \cong \operatorname{Hom}(G, B)$ it follows that each arrow in (2-4) is an isomorphism, and hence the connecting homomorphism $\operatorname{Hom}(N, B) \rightarrow \operatorname{Ext}(Q, B)$ in (2-3) is injective. On the other hand, (2) implies that $\alpha^{*}: \operatorname{Ext}(A, B) \rightarrow \operatorname{Ext}(G, B)$ is injective and therefore the arrow $\operatorname{Ext}(A, B) \rightarrow \operatorname{Ext}(Q, B)$ is an isomorphism in (2-5). This forces that $\operatorname{Hom}(N, B)=0$, as in $\left(1^{\prime}\right)$. Furthermore, $\operatorname{Ext}(N, B)$ is the cokernel of $\alpha^{*}: \operatorname{Ext}(A, B) \rightarrow \operatorname{Ext}(G, B)$, which, in view of (2), is isomorphic to $\operatorname{Hom}(B, B)$ via $\delta$. Hence $i^{*} \circ \delta$ is an isomorphism as claimed in $\left(2^{\prime}\right)$.

If $\alpha$ is surjective, then $Q=A$, so (1) follows from ( $\left(1^{\prime}\right)$. Moreover, $\left(2^{\prime}\right)$ implies that the epimorphism $i^{*}: \operatorname{Ext}(G, B) \rightarrow \operatorname{Ext}(N, B)$ splits, yielding (2). 
As a crucial example, we consider $L_{f} K(\mathbb{Z}, n)$ for an arbitrary map $f$ and $n \geq 1$. In this case, we infer from part 3 of Theorem 2.1 that $B=0$, since $\operatorname{Ext}(\mathbb{Z}, B)=0$ implies that $\operatorname{Hom}(B, B)=0$. Furthermore, if $\alpha: \mathbb{Z} \rightarrow A$ is induced by $\eta: K(\mathbb{Z}, n) \rightarrow K(A, n)$, then part 1 of Theorem 2.1 yields an isomorphism

$$
\operatorname{Hom}(A, A) \cong \operatorname{Hom}(\mathbb{Z}, A) \cong A
$$

sending each endomorphism $\varphi$ of $A$ to $\varphi(\alpha(1))$. Hence we may define a multiplication in $A$ corresponding to composition in $\operatorname{Hom}(A, A)$, and in this way $A$ acquires a ring structure with a unit element, namely $\alpha(1)$. This ring structure is of a very special kind, which we discuss next.

Definition 2.3 A ring $A$ with 1 is rigid if the evaluation map $\operatorname{Hom}(A, A) \rightarrow A$ given by $\varphi \mapsto \varphi(1)$ is bijective.

(The zero ring is viewed as a ring with $1=0$, so it is rigid.)

Theorem 2.4 For any map $f$ and any integer $n \geq 1$, we have $L_{f} K(\mathbb{Z}, n) \simeq K(A, n)$, where $A$ admits a structure of a rigid ring and the induced homomorphism $\mathbb{Z} \rightarrow A$ is a ring map. Moreover, all rigid rings occur in this way.

Proof The first assertion follows from (2-6). To prove the second claim, note that if $A$ is a rigid ring and $\alpha: \mathbb{Z} \rightarrow A$ is its unit map, then the map $f: K(\mathbb{Z}, n) \rightarrow K(A, n)$ induced by $\alpha$ is an $f$-localization.

From this fact it follows, for example, that $\pi_{n}\left(L_{f} K(\mathbb{Z}, n)\right)$ cannot be isomorphic to $\mathbb{Z} / p^{\infty}$ nor to $\mathbb{Z}[1 / p] \times \mathbb{Z}[1 / p]$. However, it can be isomorphic to $\mathbb{Z}[1 / p] \times \mathbb{Z}[1 / q]$ if $p$ and $q$ are distinct primes.

Rigid rings are also called $E$-rings, due to the fact that they satisfy $A \cong \operatorname{End}(A)$. All rigid rings are commutative (see Theorem 3.2 below for a more general result), and if $A$ is a commutative ring such that $A \cong \operatorname{End}(A)$ as rings, then it follows from Theorem 3.5 that the evaluation map $\operatorname{End}(A) \rightarrow A$ is an isomorphism, and so $A$ is rigid. This remark goes back to [70]; see also [41, Proposition 13.1.9].

The basic examples of rigid rings are the rings $\mathbb{Z} / m$, the subrings of $\mathbb{Q}$, and the ring $\widehat{\mathbb{Z}}_{p}$ of $p$-adic integers, for any $p$. If $A$ and $B$ are rigid $\operatorname{rings}$ and $\operatorname{Hom}(A, B)=0$ and $\operatorname{Hom}(B, A)=0$, then the product $A \times B$ is rigid. Other less obvious examples of rigid rings are the products

$$
\prod_{p \in P} \mathbb{Z} / p, \quad \prod_{p \in P} \mathbb{Z}_{(p)}, \quad \prod_{p \in P} \hat{\mathbb{Z}}_{p},
$$


where $P$ is an arbitrary set of primes, possibly infinite, and $\mathbb{Z}_{(p)}$ denotes the integers localized at $p$. In fact, as observed in [12, page 200], if $\left\{A_{i}\right\}_{i \in I}$ is any set of rigid rings such that $\operatorname{Hom}\left(\prod_{i \neq j} A_{i}, A_{j}\right)=0$ for all $j$, then $\prod_{i \in I} A_{i}$ is rigid. A classification of rigid rings which are torsion-free of finite rank was achieved in [60].

As shown in [30] or in [42, Chapter 30], there are rigid rings of arbitrarily large cardinality. This implies the following.

Corollary 2.5 The collection of homotopy types of the form $L_{f} S^{1}$, where $f$ ranges over all maps, is a proper class (ie not a set).

Proof According to Theorem 2.4, the distinct homotopical localizations of $S^{1}$ are in one-to-one correspondence with the rigid rings. Since, according to [30, Corollary 4.10], there is a proper class of nonisomorphic rigid rings, our claim follows.

This result is striking, since the distinct homological localizations of $S^{1}$ are listed in [7] and certainly form a set. Furthermore, Ohkawa proved in [56] that the stable Bousfield equivalence classes of spectra form a set - another proof of this fact was given later in [31]. This implies that the nonequivalent homological localization functors form a set, both in the stable and in the unstable homotopy categories, while there is a proper class of distinct $f$-localizations. We thank Neil Strickland for bringing Ohkawa's article to our attention.

The ring $A=\pi_{1}\left(L_{f} S^{1}\right)$ carries important information about the higher homotopy groups of $f$-local spaces. As we show next, this can be made particularly explicit in the case of GEMs.

Theorem 2.6 If $X$ is an $f$-local GEM for a certain map $f$ and $A$ denotes the commutative ring $\pi_{1}\left(L_{f} S^{1}\right)$, then the homotopy groups $\pi_{i}(X)$ admit $A$-module structures.

Proof If $X$ is an $f$-local GEM, then each $K\left(\pi_{i}(X), i\right)$ is $f$-local because it is a homotopy retract of $X$. Since $K\left(\pi_{i}(X), 1\right) \simeq \Omega^{i-1} K\left(\pi_{i}(X), i\right)$, each $K\left(\pi_{i}(X), 1\right)$ is $f$-local as well. Hence from the fact that $\eta: S^{1} \rightarrow K(A, 1)$ is an $f$-equivalence we obtain isomorphisms

$$
\pi_{i}(X) \cong \operatorname{Hom}\left(A, \pi_{i}(X)\right)
$$

endowing each $\pi_{i}(X)$ with an $A$-module structure.

We will show in Section 7 that the $A$-module structures stated in Theorem 2.6 are, in fact, unique. Moreover, the $A$-modules $G$ such that $G \cong \operatorname{Hom}(A, G)$ are of a 
special kind, which have also been studied in the literature [59]. Other consequences of Theorem 2.6 are discussed in Section 7.

The algebraic relations displayed in Theorem 2.1 impose strong restrictions on the group $B=\pi_{n+1}\left(L_{f} K(G, n)\right)$, which is zero in many cases, as we show next. Recall that an abelian group is called reduced if it does not contain nonzero divisible subgroups.

Theorem 2.7 For every abelian group $G$, every map $f$ and every $n \geq 1$, the group $\pi_{n+1}\left(L_{f} K(G, n)\right)$ is reduced.

Proof Write $\pi_{n+1}\left(L_{f} K(G, n)\right)=D \oplus R$ where $D$ is divisible and $R$ is reduced. Then the composite of $\eta: K(G, n) \rightarrow L_{f} K(G, n)$ with the projection onto $K(D, n+1)$ is null, since it corresponds to an element of $\operatorname{Ext}(G, D)$, which is zero since $D$ is divisible. Hence, the universal property of $\eta$ implies that $D=0$.

Theorem 2.8 For an abelian group $G$, let $L_{f} K(G, n) \simeq K(A, n) \times K(B, n+1)$ for a map $f$ and $n \geq 1$, and let $N$ be the kernel of the homomorphism $G \rightarrow A$ induced by the localization map $K(G, n) \rightarrow L_{f} K(G, n)$. If $T N$ denotes the torsion subgroup of $N$, then:

(1) There is a map $g$ such that $L_{g} K(T N, n) \simeq K(B, n+1)$.

(2) If $T N$ is reduced, then $B=0$.

Proof If we denote $F=N / T N$, then the exact sequence

$$
0 \longrightarrow T N \longrightarrow N \longrightarrow F \longrightarrow 0,
$$

together with the fact that $\operatorname{Hom}(N, B)=0$ by part $1^{\prime}$ of Lemma 2.2 , yields an exact sequence

$$
0 \longrightarrow \operatorname{Hom}(T N, B) \longrightarrow \operatorname{Ext}(F, B) \longrightarrow \operatorname{Ext}(N, B) \longrightarrow \operatorname{Ext}(T N, B) \longrightarrow 0 .
$$

Since $F$ is torsion-free, $\operatorname{Ext}(F, B)$ is divisible by [55, Theorem 4.5]. According to Theorem 2.7, the group $B$ is reduced and therefore $\operatorname{Hom}(B, B)$ is also reduced. Hence $\operatorname{Ext}(N, B)$ is reduced because it is isomorphic to $\operatorname{Hom}(B, B)$ by part $2^{\prime}$ of Lemma 2.2. It follows that the homomorphism $\operatorname{Ext}(F, B) \rightarrow \operatorname{Ext}(N, B)$ in (2-7) is zero. This has two consequences. First, since $\operatorname{Hom}(T N, B)$ is reduced and isomorphic to the divisible group $\operatorname{Ext}(F, B)$, we infer that $\operatorname{Hom}(T N, B)=0$. Second,

$$
\operatorname{Hom}(B, B) \cong \operatorname{Ext}(N, B) \cong \operatorname{Ext}(T N, B) .
$$

These two facts together imply that the composite $g$ of the arrows

$$
K(T N, n) \longrightarrow K(N, n) \longrightarrow K(G, n) \longrightarrow K(B, n+1)
$$


is a $g$-localization if $n \geq 2$. Thus $L_{g} K(T N, n) \simeq K(B, n+1)$ if $n \geq 2$.

In the case $n=1$, we need to check that, in addition, $\operatorname{Hom}\left(H_{2}(T N), B\right)=0$, as in part 4 of Theorem 2.1. This follows from the vanishing of $\operatorname{Hom}(T N, B)$, since for every abelian group $G$ there is an epimorphism $G \otimes G \rightarrow H_{2}(G)$ (see [14, Theorem V.6.4(iii)]), and $\operatorname{Hom}(G, B)=0$ implies $\operatorname{Hom}(G \otimes G, B)=0$ since there is a homomorphism from $G$ to $G \otimes G$ given by $x \mapsto x \otimes g$ for every element $g \in G$. This ends the proof of (1).

Next, we show that (1) implies (2). For each prime $p$, let $T_{p}$ be the $p$-torsion subgroup of $T N$. Then $T_{p}$ is a direct summand of $T N$, and hence $L_{g} K\left(T_{p}, n\right) \simeq$ $K(C(p), n+1)$, where $C(p) \subseteq B$. If $C(p)=0$ for all primes $p$, then $L_{g} K\left(T_{p}, n\right)$ is contractible for every $p$ and this implies, by [32, Proposition 1.D.2], that $L_{g} K(T N, n)$ is contractible, so $B=0$ as claimed. Otherwise, choose a prime $p$ such that $C(p) \neq 0$. If $K(\mathbb{Z} / p, n)$ is $g$-local, then $\operatorname{Hom}\left(T_{p}, \mathbb{Z} / p\right)=0$, and hence $p T_{p}=T_{p}$. This tells us that $T_{p}$ is $p$-divisible, and hence $\operatorname{Hom}\left(\mathbb{Z} / p^{\infty}, T_{p}\right) \neq 0$, contradicting the assumption that $T N$ is reduced. Therefore, $L_{g} K(\mathbb{Z} / p, n)$ is contractible by part 1 of Lemma 6.2, and $L_{g} K\left(\mathbb{Z} / p^{m}, n\right)$ is also contractible for every $m$ by part 2 of Lemma 6.2.

Write $T_{p}$ as a filtered colimit of its finitely generated subgroups $\left\{S_{\lambda}\right\}_{\lambda \in \Lambda}$. Since $f$-localizations preserve finite products, each $L_{g} K\left(S_{\lambda}, n\right)$ is contractible. Since homotopy groups commute with filtered homotopy colimits, $K\left(T_{p}, n\right) \simeq \operatorname{hocolim}_{\lambda \in \Lambda} K\left(S_{\lambda}, n\right)$. Using [32, Proposition 1.D.2] again, we infer that $L_{g} K\left(T_{p}, n\right)$ is contractible, and this contradicts our choice of $p$.

Corollary 2.9 Suppose that $L_{f} K(G, n) \simeq K(A, n) \times K(B, n+1)$ for an abelian group $G$, a map $f$, and $n \geq 1$. If $G$ is torsion-free or reduced, then $B=0$.

Proof Let $N$ be the kernel of the induced homomorphism $G \rightarrow A$. If $G$ is torsion-free, then $N$ is also torsion-free, and from part 1 of Theorem 2.8 it follows that $B=0$. If $G$ is reduced, then $N$ is also reduced, and part 2 of Theorem 2.8 implies that $B=0$.

The fact that $B=0$ when $G$ is reduced was first proved by Bousfield in an unpublished manuscript. We are indebted to him for passing this information on to us. His argument was outlined with permission in [43, Teorema 6.4.1] in the stable case.

Corollary 2.10 If $G$ is a finitely generated abelian group, then for every map $f$ and each integer $n \geq 1$ there is an abelian group $A$ such that $L_{f} K(G, n) \simeq K(A, n)$.

The group $B$ need not be zero if $G$ is not reduced. For example, if $L_{f}$ is ordinary homological localization with $\mathbb{Z} / p$ coefficients, then it follows from Lemma 6.1 below 
applied to the fiber sequence

$$
K(\mathbb{Z}[1 / p], n) \longrightarrow K\left(\mathbb{Z} / p^{\infty}, n\right) \longrightarrow K(\mathbb{Z}, n+1)
$$

that $L_{f} K\left(\mathbb{Z} / p^{\infty}, n\right) \simeq L_{f} K(\mathbb{Z}, n+1) \simeq K\left(\widehat{\mathbb{Z}}_{p}, n+1\right)$. This case yields an example where both $A \neq 0$ and $B \neq 0$, namely

$$
L_{f} K\left(\mathbb{Z} \oplus \mathbb{Z} / p^{\infty}, n\right) \simeq K\left(\widehat{\mathbb{Z}}_{p}, n\right) \times K\left(\widehat{\mathbb{Z}}_{p}, n+1\right) .
$$

Theorem 2.11 Let $G$ be any abelian group, $f$ any map, and $n \geq 1$. If $L_{f} K(G, n) \simeq$ $K(A, n) \times K(B, n+1)$, then there is a torsion divisible direct summand $T \subseteq G$ such that $L_{f} K(T, n) \simeq K(B, n+1)$ and $\operatorname{Hom}(B, B) \cong \operatorname{Ext}(T, B)$.

Proof Write $G \cong D \oplus R$ where $D$ is divisible and $R$ is reduced, and let $T D$ denote the torsion subgroup of $D$. Thus, $D \cong T D \oplus F$ where $F$ is torsion-free. Now Corollary 2.9 tells us that $\pi_{n+1}\left(L_{f} K(F, n)\right)=0$ and $\pi_{n+1}\left(L_{f} K(R, n)\right)=0$. Therefore, since $L_{f}$ preserves finite products, we conclude that $L_{f} K(T D, n) \simeq K\left(A^{\prime}, n\right) \times K(B, n+1)$ for some $A^{\prime} \subseteq A$.

Let $P$ denote the set of primes $p$ such that $K\left(\mathbb{Z} / p^{\infty}, n\right)$ is $f$-local, and let $P^{\prime}$ denote the complementary set of primes. Let $L$ be the $P$-torsion subgroup of $T D$ and let $T$ be the $P^{\prime}$-torsion subgroup, so $T D \cong L \oplus T$. Thus, by the structure theorem of divisible abelian groups [61, Theorem 4.1.5], $L \cong \bigoplus_{p \in P} \bigoplus_{\kappa_{p}} \mathbb{Z} / p^{\infty}$ for some cardinals $\kappa_{p}$. This embeds into $\prod_{p \in P} \prod_{\kappa_{p}} \mathbb{Z} / p^{\infty}$, and hence is a direct summand in it, since every divisible subgroup splits. Therefore, $K(L, n)$ is $f$-local since it is a retract of a product of $f$-local spaces. Hence, $L_{f} K(T D, n) \simeq K(L, n) \times L_{f} K(T, n)$ and $L_{f} K(T, n) \simeq K\left(A^{\prime \prime}, n\right) \times K(B, n+1)$ for some $A^{\prime \prime} \subseteq A^{\prime}$. Suppose that $A^{\prime \prime} \neq 0$ and let $\alpha: T \rightarrow A^{\prime \prime}$ be the homomorphism induced by the localization map, which is nonzero by part 1 of Theorem 2.1. So there is a prime $p \in P^{\prime}$ and a direct summand $S \subseteq T$ isomorphic to $\mathbb{Z} / p^{\infty}$ whose image in $A^{\prime \prime}$ is nonzero. Then $K\left(\mathbb{Z} / p^{\infty}, n\right)$ is a retract of $K\left(A^{\prime \prime}, n\right)$, and this implies that $K\left(\mathbb{Z} / p^{\infty}, n\right)$ is $f$-local, contradicting the fact that $p \in P^{\prime}$. This proves that $A^{\prime \prime}=0$, as we wanted to show. The isomorphism $\operatorname{Hom}(B, B) \cong \operatorname{Ext}(T, B)$ follows from part 3 of Theorem 2.1.

Corollary 2.12 Let a map $f$, a prime $p$ and an integer $n \geq 1$ be given. Then either $K\left(\mathbb{Z} / p^{\infty}, n\right)$ is $f$-local or $L_{f} K\left(\mathbb{Z} / p^{\infty}, n\right) \simeq K(B, n+1)$, where $B$ satisfies

$$
\operatorname{Hom}(B, B) \cong \operatorname{Ext}\left(\mathbb{Z} / p^{\infty}, B\right) .
$$

For a set of primes $P$, an abelian group $B$ is called Ext- $P$-complete if

$$
\operatorname{Hom}\left(\mathbb{Z}\left[P^{-1}\right], B\right)=0=\operatorname{Ext}\left(\mathbb{Z}\left[P^{-1}\right], B\right),
$$


or, equivalently, if the natural homomorphism

$$
\left.B \cong \operatorname{Hom}(\mathbb{Z}, B) \longrightarrow \operatorname{Ext}\left(\mathbb{Z}\left[P^{-1}\right] / \mathbb{Z}\right), B\right) \cong \prod_{p \in P} \operatorname{Ext}\left(\mathbb{Z} / p^{\infty}, B\right)
$$

is an isomorphism. Thus, for a single prime $p$, an abelian group $B$ is Ext- $p$-complete if the natural homomorphism $B \rightarrow \operatorname{Ext}\left(\mathbb{Z} / p^{\infty}, B\right)$ is an isomorphism. The only examples that we know of abelian groups $B$ such that $\operatorname{Hom}(B, B) \cong \operatorname{Ext}\left(\mathbb{Z} / p^{\infty}, B\right)$ are the rigid rings whose underlying abelian group is Ext- $p$-complete. According to [12, Corollary 3.5], these are necessarily isomorphic to $\widehat{\mathbb{Z}}_{p}$ or $\mathbb{Z} / p^{k}$ for some $k$.

\section{Rigid rings and rigid algebras}

In this section, all rings are assumed to be associative and have an identity element, which we denote by 1 if no confusion can arise.

Recall from Section 2 that a ring $A$ is called rigid if the evaluation map $\operatorname{Hom}(A, A) \rightarrow A$ given by $\varphi \mapsto \varphi(1)$ is bijective. We shall, in fact, discuss a more general notion, namely rigid algebras. Most of the following results generalize basic properties of rigid rings that can be found in $[12 ; 70]$. Some observations are new, notably Theorem 3.3. A more detailed study of rigid algebras and their modules was undertaken by Strüngmann in [72]. Rigid $R$-algebras are also called $E(R)$-algebras elsewhere. The book [41] by Göbel and Trlifaj contains a chapter devoted to them (Chapter 13), and they are discussed in greater detail in [42].

In the rest of this section, $R$ will be supposed to be a commutative ring. By an $R$-algebra we mean a ring $A$ equipped with a central ring homomorphism $R \rightarrow A$.

Definition 3.1 An $R$-algebra $A$ will be called rigid if the evaluation map

$$
\operatorname{Hom}_{R}(A, A) \longrightarrow A
$$

given by $\varphi \mapsto \varphi(1)$ is bijective.

Theorem 3.2 If an $R$-algebra $A$ is rigid, then $A$ is commutative.

Proof Fix any element $a \in A$. Then the $R$-endomorphisms $\varphi_{1}$ and $\varphi_{2}$ of $A$ given by

$$
\varphi_{1}(x)=a x \quad \text { and } \quad \varphi_{2}(x)=x a
$$

satisfy $\varphi_{1}(1)=\varphi_{2}(1)$, and hence are equal. 
If $A$ is any $R$-algebra, then left multiplication defines a map $\mu: A \rightarrow \operatorname{Hom}_{R}(A, A)$. Both $\mu$ and the evaluation map $\varepsilon: \operatorname{Hom}_{R}(A, A) \rightarrow A$ are $R$-module homomorphisms and the composition $\varepsilon \circ \mu$ is the identity map. Therefore, $\varepsilon$ is surjective and $\mu$ is injective for every $R$-algebra $A$. It follows that an $R$-algebra is rigid if and only if the evaluation map $\varepsilon$ is injective.

Theorem 3.3 An $R$-algebra $A$ is rigid if and only if the underlying $R$-module admits only one compatible multiplication where 1 acts as a left identity.

Proof Suppose first that $A$ is rigid, and denote by $\circ$ an arbitrary multiplication in $A$ which is compatible with the $R$-module structure and where $1 \circ a=a$ for all $a$. Then, for any fixed element $a \in A$, the $R$-endomorphisms $\varphi_{1}$ and $\varphi_{2}$ given by $\varphi_{1}(x)=x a$ and $\varphi_{2}(x)=x \circ a$ satisfy $\varphi_{1}(1)=\varphi_{2}(1)$ and hence are equal. This proves one implication.

Conversely, suppose that the multiplication in $A$ is unique with the prescribed conditions. If $\psi$ is an $R$-endomorphism of $A$ such that $\psi(1)=1$, then the multiplication defined by $a \circ b=\psi(a) b$ endows $A$ with an $R$-algebra structure where 1 is a left identity. By assumption, $a \circ b=a b$ for all $a, b \in A$, which implies that $\psi=\mathrm{id}$. Now, if $\varphi_{1}$ and $\varphi_{2}$ are two $R$-endomorphisms of $A$ such that $\varphi_{1}(1)=\varphi_{2}(1)$, then $\psi=\mathrm{id}-\varphi_{1}+\varphi_{2}$ satisfies $\psi(1)=1$, and hence $\varphi_{1}=\varphi_{2}$. This proves that $A$ is rigid, as claimed.

Example 3.4 The abelian group $\mathbb{Z} \oplus \mathbb{Z}$ admits a two-parameter family of distinct multiplications for which $(1,1)$ is a two-sided identity. Each of these is determined by a $2 \times 2$ matrix with integer entries, representing left multiplication by $(1,0)$ in $\mathbb{Z} \oplus \mathbb{Z}$. Thus, if we impose the condition that the product of this matrix with $(1,1)$ equals $(1,0)$, we obtain the family of solutions

$(x, y) \circ(z, t)=(\lambda x z+(1-\lambda) x t+(1-\lambda) y z-(1-\lambda) y t, \mu x z-\mu x t-\mu y z+(1+\mu) y t)$,

where $\lambda$ and $\mu$ are arbitrary integers. These multiplications are all associative and commutative.

The following theorem extends results proved by Schultz [70] in the case $R=\mathbb{Z}$. Essentially the same statements can be found in [41, Proposition 13.1.9]. We supply a proof for completeness.

Theorem 3.5 For an $R$-algebra $A$, the following statements are equivalent:

(1) $A$ is rigid.

(2) The map $\mu: A \rightarrow \operatorname{Hom}_{R}(A, A)$ given by $\mu(a)(x)=a x$ is bijective. 
(3) $\operatorname{Hom}_{R}(A /\langle 1\rangle, A)=0$, where $\langle 1\rangle$ is the $R$-submodule of $A$ generated by 1 .

(4) Every $\varphi \in \operatorname{Hom}_{R}(A, A)$ is an $A$-module endomorphism.

(5) The evaluation map $\varepsilon: \operatorname{Hom}_{R}(A, A) \rightarrow A$ is an isomorphism of $R$-algebras.

(6) The endomorphism ring $\operatorname{Hom}_{R}(A, A)$ is commutative.

Proof The equivalence of (1) and (2) follows from the fact that $\mu$ is right inverse to $\varepsilon$. Next, observe that the inclusion of the submodule $\langle 1\rangle$ into $A$ gives rise to a short exact sequence of $R$-modules

$$
0 \longrightarrow \operatorname{Hom}_{R}(A /\langle 1\rangle, A) \longrightarrow \operatorname{Hom}_{R}(A, A) \longrightarrow \operatorname{Hom}_{R}(\langle 1\rangle, A) \longrightarrow 0,
$$

where the third arrow coincides with the evaluation map $\varepsilon$, and hence it is surjective. This proves that (1) and (3) are equivalent. Next we prove that (1) $\Longrightarrow(4)$. Let $\varphi$ be an $R$-endomorphism of $A$. Fix any element $a \in A$. Then the endomorphisms $\varphi_{1}$ and $\varphi_{2}$ given by

$$
\varphi_{1}(x)=x \varphi(a) \quad \text { and } \quad \varphi_{2}(x)=\varphi(x a)
$$

satisfy $\varphi_{1}(1)=\varphi_{2}(1)$ and hence are equal. This shows that $\varphi$ is an $A$-module endomorphism, as required. The implication (4) $\Longrightarrow(1)$ is immediate since, under (4), any $\varphi \in \operatorname{Hom}_{R}(A, A)$ is completely determined by its value on 1 . We can now infer that $(4) \Longrightarrow(5)$, since

$$
\varepsilon(\psi \circ \varphi)=\psi(\varphi(1))=\varphi(1) \psi(1)=\psi(1) \varphi(1)=\varepsilon(\psi) \varepsilon(\varphi) .
$$

The fact that $(5) \Longrightarrow$ (6) follows from Theorem 3.2, and we conclude by showing that $(6) \Longrightarrow(4)$. Thus, assume that $\operatorname{Hom}_{R}(A, A)$ is a commutative ring, and pick any $\varphi \in \operatorname{Hom}_{R}(A, A)$. Then, by assumption, $\varphi$ commutes with $\mu(a)$ for any $a \in A$, which yields

$$
\varphi(a x)=[\varphi \circ \mu(a)](x)=[\mu(a) \circ \varphi](x)=a \varphi(x)
$$

for all $x \in A$, as we wanted to prove.

Recall from [11] that a ring $A$ with 1 is called solid if the multiplication map

$$
m: A \otimes A \longrightarrow A, \quad m(a \otimes b)=a b,
$$

is bijective. Such rings were called T-rings in [12] and $\mathbb{Z}$-epimorphs in [25]. Indeed, by [71, Proposition XI.1.2], a ring $A$ is solid if and only if the unit map $\mathbb{Z} \rightarrow A$ is an epimorphism of rings.

More generally, if $R$ is any commutative ring, an $R$-algebra $A$ will be called solid if the multiplication map $m: A \otimes_{R} A \rightarrow A$ is bijective or, equivalently, if the structure map $R \rightarrow A$ is an epimorphism of rings. 
The fact that an $R$-algebra $A$ is solid forces that $a \otimes b=a b \otimes 1=1 \otimes a b$ in $A \otimes_{R} A$, for all $a$ and $b$. Therefore, if $A$ is solid, then, for every $\varphi \in \operatorname{Hom}_{R}(A, A)$, we consider the homomorphism $\Phi: A \otimes_{R} A \rightarrow A$ given by $\Phi(a \otimes b)=a \varphi(b)$ and infer that

$$
\varphi(a x)=\Phi(1 \otimes a x)=\Phi(a \otimes x)=a \varphi(x) .
$$

Hence, every $\varphi \in \operatorname{Hom}_{R}(A, A)$ is an $A$-module endomorphism, and Theorem 3.5 yields the following result, which generalizes [12, Corollary 1.8].

\section{Theorem 3.6 Every solid $R$-algebra is rigid.}

The following comparison makes the distinction between solid $R$-algebras and rigid $R$-algebras clearer. By definition, an $R$-algebra $A$ is solid if and only if the structure map $R \rightarrow A$ is a ring epimorphism, and $A$ is rigid if and only if the structure map $R \rightarrow A$ is a localization, in the sense that every $R$-module homomorphism from $R$ to $A$ can be uniquely extended to an endomorphism of $A$. Such "discrete localizations" are analyzed further in the next sections.

The $p$-adic integer ring is rigid as a $\mathbb{Z}$-algebra, but not solid. Solid rings have been classified in $[11 ; 12 ; 25 ; 76]$. As stated in [12, Proposition 1.10$]$, a ring $A$ is solid if and only if $A / T(A)$ is isomorphic to a subring of $\mathbb{Q}$ and, if $p$ is a prime such that $T_{p}(A) \neq 0$, then $T_{p}(A)$ is cyclic and $A / T(A)$ is $p$-divisible. Here $T(A)$ denotes the torsion subgroup of $A$ and $T_{p}(A)$ is its $p$-primary component.

Proposition 3.7 The solid rings are precisely the rigid rings of rank at most 1 .

Proof The fact that solid rings have rank at most 1 is deduced from their classification. Conversely, according to [70, Lemmas 2 and 3], if $A$ is rigid then for every prime $p$ the group $T_{p}(A)$ is cyclic and $A=T_{p}(A) \oplus A_{p}$, where $A_{p}$ is $p$-divisible.

We warn the reader that, while the class of solid rings is closed under quotients, the class of rigid rings is not. For example, the quotient of $A=\mathbb{Z}\left[\frac{1}{2}\right] \times \mathbb{Z}\left[\frac{1}{3}\right]$ by the ideal $5 A$ is isomorphic to $\mathbb{Z} / 5 \times \mathbb{Z} / 5$.

\section{Algebraic structures preserved by localizations}

In this section, we deal with localization in the category of groups with respect to a group homomorphism $\varphi: W \rightarrow V$, as in [18, Section 3] or [23, Section 1]. A group $X$ is said to be $\varphi$-local if the induced map

$$
\operatorname{Hom}(\varphi, X): \operatorname{Hom}(V, X) \longrightarrow \operatorname{Hom}(W, X)
$$


is a bijection of sets. A $\varphi$-equivalence of groups is a homomorphism $\psi$ such that $\operatorname{Hom}(\psi, X)$ is a bijection for every $\varphi$-local group $X$. Since the category of groups is locally presentable [1], for every group $G$ there is a $\varphi$-equivalence $\eta_{G}: G \rightarrow L_{\varphi} G$ into a $\varphi$-local group $L_{\varphi} G$, with universal properties analogous to those mentioned in Section 1 ; thus $\left(L_{\varphi}, \eta\right)$ is an idempotent monad on the category of groups. We call $L_{\varphi} G$ the $\varphi$-localization of $G$.

It is well known that every localization of an abelian group is abelian. The following argument is due to Farjoun.

Proposition 4.1 Let $(L, \eta)$ be any idempotent monad on the category of groups. If $A$ is any abelian group, then $L A$ is also abelian.

Proof For any element $a \in A$, conjugation by $\eta_{A}(a)$ is the identity homomorphism on $\eta_{A}(A)$, and hence it is the identity homomorphism on $L A$. In particular, for each $x \in L A$, conjugation by $x$ is the identity on $\eta_{A}(A)$, and hence it is the identity on $L A$. This shows that $L A$ is indeed abelian.

The following consequence is analogous to part 4 of Theorem 1.3.

Proposition 4.2 Let $\varphi: W \rightarrow V$ be a group homomorphism and $\varphi_{\mathrm{ab}}: W_{\mathrm{ab}} \rightarrow V_{\mathrm{ab}}$ its abelianization. Then there is a natural isomorphism $L_{\varphi} A \cong L_{\varphi_{\mathrm{ab}}} A$ for every abelian group $A$.

Proof Since both $L_{\varphi}$ and $L_{\varphi_{\mathrm{ab}}}$ send abelian groups to abelian groups, it suffices to observe - directly from the definition - that an abelian group is $\varphi_{\mathrm{ab}}$-local if and only if it is $\varphi$-local.

In the rest of this section, we fix a (not necessarily commutative) ring $R$ with 1 and an arbitrary idempotent monad $(L, \eta)$ on the category of abelian groups. Most of the results given below are part of the $\mathrm{Ph} \mathrm{D}$ thesis of the second-named author [62], and some of them were also collected in [41, Section 13.1]. However, here we remove the unnecessary assumption that the ring $R$ be commutative.

If $A$ is a ring or a module over some ring, we denote by $L A$ the localization of the underlying abelian group. Since the functor $L$ is left adjoint to an additive functor, it follows that $L$ itself is additive [50, page 83]; that is, the natural map

$$
\operatorname{Hom}(A, B) \longrightarrow \operatorname{Hom}(L A, L B)
$$

is a group homomorphism for all abelian groups $A$ and $B$. In the case when $A=B$, this map is in fact a ring homomorphism (under composition). Thus, if $M$ is a left 
$R$-module with structure map $R \rightarrow \operatorname{Hom}(M, M)$, then $L M$ inherits a left $R$-module structure such that $\eta_{M}: M \rightarrow L M$ is an $R$-module map, by composing

$$
R \longrightarrow \operatorname{Hom}(M, M) \longrightarrow \operatorname{Hom}(L M, L M) .
$$

Moreover, the $R$-module structure on $L M$ is unique if we impose the condition that $\eta_{M}$ be an $R$-module map, since each endomorphism $r: M \rightarrow M$ induces a unique $\tilde{r}: L M \rightarrow L M$ such that $\tilde{r} \circ \eta_{M}=\eta_{M} \circ r$ by the universal property of $L$. Thus we have proved the following.

Theorem 4.3 If $M$ is a left $R$-module, then $L M$ admits a unique left $R$-module structure such that the localization map $\eta_{M}: M \rightarrow L M$ is an $R$-module map.

There are other ways of proving the same result. Note that $T A=R \otimes A$ defines a monad on abelian groups, whose algebras are precisely the left $R$-modules. Then Theorem 1.2 applies to yield another proof of Theorem 4.3. The fact that $T$ preserves $L$-equivalences is a direct consequence of the hom-tensor adjunction. More generally, the following is true.

Lemma 4.4 The tensor product of any two $L$-equivalences is an $L$-equivalence.

Proof If $f: A \rightarrow B$ and $g: C \rightarrow D$ are $L$-equivalences, and $Y$ is $L$-local, then $f$ and $g$ induce isomorphisms

$$
\begin{aligned}
\operatorname{Hom}(B \otimes D, Y) & \cong \operatorname{Hom}(B, \operatorname{Hom}(D, Y)) \cong \operatorname{Hom}(B, \operatorname{Hom}(C, Y)) \\
& \cong \operatorname{Hom}(C, \operatorname{Hom}(B, Y)) \cong \operatorname{Hom}(C, \operatorname{Hom}(A, Y)) \\
& \cong \operatorname{Hom}(A \otimes C, Y),
\end{aligned}
$$

so $f \otimes g$ is also an $L$-equivalence.

Corollary 4.5 If $F$ is a field and $V$ is a vector space over $F$, then $L V=0$ or $L V \cong V$.

Proof By Theorem 4.3, $L V$ is a vector space over $F$ and hence isomorphic to a direct sum of copies of $F$. Since every retract of an $L$-local group is $L$-local, it follows that $F$ is $L$-local (unless $L V=0$ ) and hence $V$ is $L$-local as well, since it is a retract of a product of copies of $F$.

Lemma 4.6 If $M$ is any $R$-module, then the natural map $\operatorname{Hom}_{R}(L R, L M) \rightarrow L M$ induced by the localization map $\eta_{R}: R \rightarrow L R$ is an isomorphism. 
Proof The universal property of $L$ gives rise to an isomorphism of abelian groups $\operatorname{Hom}(L R, L M) \cong \operatorname{Hom}(R, L M)$, which restricts to a monomorphism

$$
\operatorname{Hom}_{R}(L R, L M) \longrightarrow \operatorname{Hom}_{R}(R, L M) \cong L M .
$$

Now, given an $R$-module map $\psi: R \rightarrow L M$, it follows again from the universal property of $L$ that the induced homomorphism $\tilde{\psi}: L R \rightarrow L M$ is an $R$-module map, since

$$
\widetilde{\psi}\left(r \eta_{R}(s)\right)=\tilde{\psi}\left(\eta_{R}(r s)\right)=\psi(r s)=r \psi(s)=r \tilde{\psi}\left(\eta_{R}(s)\right)
$$

for all $r, s \in R$. This shows that (4-1) is, in fact, bijective.

Theorem 4.7 If $R$ is a ring, then $L R$ admits a unique ring structure such that the localization map $\eta_{R}: R \rightarrow L R$ is a ring homomorphism. If $R$ is commutative, then $L R$ is commutative and it is rigid as an $R$-algebra.

Proof We can use (4-1) with $M=R$ to endow $L R$ with a ring structure, where the multiplication is induced by composition in $\operatorname{Hom}_{R}(L R, L R)$. It follows from this definition that $\eta_{R}$ is a ring homomorphism, and, if $R$ is commutative, then $L R$ is rigid as an $R$-algebra. As such, the multiplication in $L R$ is commutative by Theorem 3.2 and unique by Theorem 3.3.

If $R$ is not necessarily commutative, then the uniqueness of the multiplication can be inferred from Lemma 4.4, since a ring structure on $L R$ can be viewed as an abelian group homomorphism $L R \otimes L R \rightarrow L R$, and there is only one compatible with the multiplication $R \otimes R \rightarrow R$, since

$$
\eta_{R} \otimes \eta_{R}: R \otimes R \longrightarrow L R \otimes L R
$$

is an $L$-equivalence.

A similar strategy can be used to prove other results, such as the following one.

Lemma 4.8 If $f: R \rightarrow S$ is a ring homomorphism, then $L f: L R \rightarrow L S$ is also a ring homomorphism.

Proof If $\mu_{R}: R \otimes R \rightarrow R$ and $\mu_{S}: S \otimes S \rightarrow S$ denote the respective multiplications, then $L f$ is a ring homomorphism if and only if $L f \circ \mu_{R}=\mu_{S} \circ(L f \otimes L f)$. This is checked by composing with $\eta_{R} \otimes \eta_{R}$ on the right and next using, once more, the universal property of $L$. 
As a consequence, it follows that if $R$ is commutative and $A$ is any $R$-algebra (ie a ring homomorphism $f: R \rightarrow A$ ), then $L A$ admits not only a unique $R$-algebra structure such that $\eta_{A}: A \rightarrow L A$ is a homomorphism of $R$-algebras, but also a unique compatible $L R$-algebra structure given by the ring homomorphism $L f: L R \rightarrow L A$. As shown next, the same happens with $R$-modules.

Theorem 4.9 If $M$ is a left $R$-module, then the $R$-module structure of $L M$ can be extended uniquely to a left $L R$-module structure.

Proof For this, use that $\eta_{R} \otimes \eta_{M}: R \otimes M \rightarrow L R \otimes L M$ is an $L$-equivalence.

We conclude this section with a less straightforward consequence of Theorem 4.3.

Theorem 4.10 If $R=\mathbb{Z} / p^{r}$ for some prime $p$ and $r \geq 1$, then $L R \cong \mathbb{Z} / p^{i}$ for some $i \leq r$, and $\eta_{R}: R \rightarrow L R$ is mod $p^{i}$ reduction. Moreover, all exponents $i \leq r$ can occur.

Proof By Theorem 4.3, $L R$ has an $R$-module structure, and hence it is annihilated by $p^{r}$. Since every abelian group of finite exponent is a direct sum of cyclic groups (as shown in [48, Theorem 6] or [61, Theorem 4.3.5]), $L R$ is isomorphic to a direct sum of copies of $\mathbb{Z} / p^{j}$ with $j \leq r$. Since $\eta_{R}: R \rightarrow L R$ induces an isomorphism

$$
\operatorname{Hom}(L R, L R) \cong \operatorname{Hom}(R, L R),
$$

we infer that $L R \cong \mathbb{Z} / p^{i}$ for some $i \leq r$ and $\eta_{R}$ is indeed $\bmod p^{i}$ reduction. To prove the last claim, note that, if $L$ is localization with respect to the projection $\mathbb{Z} / p^{r} \rightarrow \mathbb{Z} / p^{i}$, then $L\left(\mathbb{Z} / p^{r}\right) \cong \mathbb{Z} / p^{i}$.

\section{Relating discrete and homotopical localizations}

Let $G$ be any abelian group and $n \geq 1$. Then, as discussed in Section 1 , for any map $f$, the $f$-localization of a $K(G, n)$ takes the form

$$
\eta: K(G, n) \longrightarrow K(A, n) \times K(B, n+1) .
$$

If we denote by $\alpha: G \rightarrow A$ the homomorphism induced by $\eta$ on the $n^{\text {th }}$ homotopy group, then part 1 of Theorem 2.1 says precisely that the group $A$ is $\alpha$-local. Since $\alpha$ is, of course, an $\alpha$-equivalence, the group $A$ is the $\alpha$-localization of $G$. Moreover, part 2 of Theorem 2.1 tells us that $B$ is $\alpha$-local as well. Therefore: 
Theorem 5.1 Given any abelian group $G$, any $n \geq 1$ and any map $f$, there exists a group homomorphism $\alpha$ such that

$$
L_{f} K(G, n) \simeq K\left(L_{\alpha} G, n\right) \times K(B, n+1),
$$

and the group $B$ is $\alpha$-local.

This is relevant because the group $A=\pi_{n}\left(L_{f} K(G, n)\right)$ therefore inherits from $G$ all the properties that are preserved by idempotent monads on abelian groups.

Theorem 5.1 can be improved if the source and target of $f$ are $(n-1)$-connected spaces. In that case, as stated in Theorem 5.4 below, the homomorphism $\alpha$ can be chosen to be equal to $\pi_{n}(f)$.

First of all, observe that, if $f$ is a map between $(n-1)$-connected spaces, then the localization $P_{S^{n}}$ with respect to $S^{n} \rightarrow *$ (ie the $n-1$ Postnikov section) trivially turns $f$ into a homotopy equivalence. This implies that, for all spaces $X$, the $f$-localization map $X \rightarrow L_{f} X$ induces a homotopy equivalence $P_{S^{n}} X \simeq P_{S^{n}} L_{f} X$; see [73, Section 3]. From this fact we derive a generalization of [8, Corollary 4.4] and [73, Section 8]. We are thankful to Jeff Smith for making this result evident to us.

Theorem 5.2 Let $f: W \rightarrow V$ be any map in which $W$ and $V$ are $(n-1)$-connected spaces. Then, for all connected spaces $X$, the natural map $X\langle n-1\rangle \rightarrow\left(L_{f} X\right)\langle n-1\rangle$ of $(n-1)$-connected covers is an $f$-localization; that is, it induces a homotopy equivalence

$$
L_{f}(X\langle n-1\rangle) \simeq\left(L_{f} X\right)\langle n-1\rangle .
$$

Proof Apply fiberwise $f$-localization [32, Section 1.F] to the homotopy fibration

$$
X\langle n-1\rangle \longrightarrow X \longrightarrow P_{S^{n}} X,
$$

thus obtaining a homotopy fibration

$$
L_{f}(X\langle n-1\rangle) \longrightarrow Y \longrightarrow P_{S^{n}} X
$$

together with a map $h: X \rightarrow Y$ which is an $f$-equivalence; see [32, Theorem 1.F.1]. Since, by our assumption, the mapping $\operatorname{spaces}_{\operatorname{map}_{*}}\left(V, P_{S^{n}} X\right)$ and $\operatorname{map}_{*}\left(W, P_{S^{n}} X\right)$ are weakly contractible, we infer that $\operatorname{map}_{*}(V, Y) \rightarrow \operatorname{map}_{*}(W, Y)$ is a weak homotopy equivalence, and hence $Y$ is $f$-local. This means, of course, that $Y \simeq L_{f} X$. Since $P_{S^{n}} X \simeq P_{S^{n}} L_{f} X$, our claim follows.

Using this observation and the same arguments as in [18, Proposition 3.3], we find that for an arbitrary map $f: W \rightarrow V$ between $(n-1)$-connected spaces, if we denote by $\varphi: \pi_{n}(W) \rightarrow \pi_{n}(V)$ the induced homomorphism of $n^{\text {th }}$ homotopy groups, then the following hold: 
(1) A group $G$ is $\varphi$-local if and only if a $K(G, n)$ is $f$-local.

(2) If $g$ is any $f$-equivalence of connected spaces, then the homomorphism $\pi_{n}(g)$ is a $\varphi$-equivalence of groups.

(To prove (2), notice that if $g$ is an $f$-equivalence, then so is the lifting of $g$ to the $(n-1)$-connected covers, by Theorem 5.2.)

In particular, since $\eta_{X}: X \rightarrow L_{f} X$ is an $f$-equivalence, it follows from (2) that for every connected space $X$ there is a natural homomorphism

$$
\pi_{n}\left(L_{f} X\right) \longrightarrow L_{\varphi} \pi_{n}(X)
$$

which is a $\varphi$-equivalence, and therefore it is an isomorphism if and only if $\pi_{n}\left(L_{f} X\right)$ is $\varphi$-local. This yields the following improvement of [23, Theorem 2.1]:

Theorem 5.3 Let $f: W \rightarrow V$ be a map where $W$ is a wedge of copies of $S^{n}$ with $n \geq 1$, and $V$ has one 0 -cell and further cells in dimensions $n$ and $n+1$ only. Let $\varphi=\pi_{n}(f)$. Then $\pi_{n}\left(L_{f} X\right) \cong L_{\varphi} \pi_{n}(X)$ for all connected spaces $X$.

Proof We only need to prove that $\pi_{n}\left(L_{f} X\right)$ is $\varphi$-local. The assumption made on $W$ ensures that, given any group homomorphism $\psi: \pi_{n}(W) \rightarrow \pi_{n}\left(L_{f} X\right)$, there exists a map $g$ : $W \rightarrow L_{f} X$ inducing $\psi$ on the $n^{\text {th }}$ homotopy groups. Since $L_{f} X$ is $f$-local, there is a map $g^{\prime}: V \rightarrow L_{f}(X)$ such that $g^{\prime} \circ f \simeq g$, yielding a homomorphism $\psi^{\prime}: \pi_{n}(V) \rightarrow \pi_{n}\left(L_{f} X\right)$ such that $\psi^{\prime} \circ \varphi=\psi$, as desired. If $\psi^{\prime \prime}$ is any other homomorphism with this property, then it is induced by some map $g^{\prime \prime}: V \rightarrow L_{f} X$. Then $g^{\prime \prime} \circ f$ and $g$ induce the same homomorphism on the $n^{\text {th }}$ homotopy groups and hence they are homotopic, since $W$ is a wedge of copies of $S^{n}$. It follows that $g^{\prime \prime} \simeq g^{\prime}$, and therefore $\psi^{\prime \prime}=\psi^{\prime}$, as needed.

Theorem 5.4 For any abelian group $G$ and any map $f$ between $(n-1)$-connected spaces, where $n \geq 1$, we have

$$
L_{f} K(G, n) \simeq K\left(L_{\varphi} G, n\right) \times K(B, n+1),
$$

where $\varphi=\pi_{n}(f)$. Moreover, the group $B$ is $\varphi$-local.

Proof Let $A$ and $B$ be the homotopy groups of $L_{f} K(G, n)$. Then the localization map $\eta: K(G, n) \rightarrow L_{f} K(G, n)$ induces a homomorphism $\pi_{n}(\eta): G \rightarrow A$. Since the map $\eta$ is an $f$-equivalence, the homomorphism $\pi_{n}(\eta)$ is a $\varphi$-equivalence. Moreover, the space $K(A, n)$ is $f$-local, and hence the group $A$ is $\varphi$-local. This proves that $A \cong L_{\varphi} G$. From the fact that $K(B, n+1)$ is $f$-local, it follows that $K(B, n)$ is also $f$-local, and therefore the group $B$ is $\varphi$-local. 
As a consequence of Proposition 4.2, we can replace $L_{\varphi} G$ with $L_{\phi} G$ in Theorem 5.4, where $\phi=H_{n}(f)$, for any $n \geq 1$.

Corollary 5.5 Suppose that $f$ is a map between $(n-1)$-connected spaces such that the homomorphism $\pi_{n}(f)$ is surjective. Then, for any abelian group $G$, the natural homomorphism $G \rightarrow \pi_{n}\left(L_{f} K(G, n)\right)$ is surjective.

Proof This follows from the fact that, if $\varphi$ is an epimorphism, then the localization map $\eta: G \rightarrow L_{\varphi} G$ is an epimorphism for all groups $G$. To prove this claim, check that the image of $\eta$ is $\varphi$-local, and its inclusion in $L_{\varphi} G$ is a $\varphi$-equivalence and hence an isomorphism.

The next example shows that the assumption that $f$ is a map between $(n-1)$-connected spaces cannot be removed from Theorem 5.4 and Corollary 5.5. Consider the map $f: M(\mathbb{Z}[1 / p], 1) \rightarrow *$, where $M$ stands for a Moore space. Then $\pi_{n}\left(L_{f} K(\mathbb{Z}, n)\right)$ is the ring $\widehat{\mathbb{Z}}_{p}$ of $p$-adic integers if $n \geq 2$; see [23]. However, any homomorphism induced by $f$ on homotopy groups will be surjective, and $\widehat{\mathbb{Z}}_{p}$ cannot be obtained by localizing $\mathbb{Z}$ with respect to any epimorphism.

Example 5.6 Let $f: X \rightarrow Y$ be any map inducing the projection $\phi: \mathbb{Z} \rightarrow \mathbb{Z} / m$ on the first homology group, where $m$ is any integer. Then an abelian group $A$ is $\phi$-local if and only if $m A=0$. Therefore,

$$
\pi_{1}\left(L_{f} K(G, 1)\right) \cong L_{\phi} G \cong G / m G
$$

for every abelian group $G$. In fact, we shall see in Corollary 7.2 that $\pi_{2}\left(L_{f} K(G, 1)\right)$ vanishes, and hence $L_{f} K(G, 1) \simeq K(G / m G, 1)$.

Similarly, if $g: X \rightarrow Y$ is any map with $H_{1}(X)=0$ and $H_{1}(Y) \cong \mathbb{Z} / p^{r}$ for a prime $p$ and $r \geq 1$, then $\pi_{1}\left(L_{g} K(G, 1)\right) \cong G / T_{p} G$ for any abelian group $G$, where $T_{p} G$ denotes the $p$-torsion subgroup of $G$.

Next, we specialize to the case $G=\mathbb{Z}$. Let $f$ be any map between connected spaces. Let $\varphi=\pi_{1}(f)$ be the homomorphism induced by $f$ on fundamental groups, and denote by $\phi=H_{1}(f)$ its abelianization. The following result follows from Theorem 2.1 and Proposition 4.2.

Theorem 5.7 For any given map $f$ between connected spaces, we have

$$
L_{f} S^{1} \simeq K\left(L_{\varphi} \mathbb{Z}, 1\right) \simeq K\left(L_{\phi} \mathbb{Z}, 1\right),
$$

where $\varphi=\pi_{1}(f)$ and $\phi=H_{1}(f)$. 
Corollary 5.8 Suppose that $H_{1}(f)$ is surjective. Then $\pi_{1}\left(L_{f} S^{1}\right)$ is cyclic.

The next result summarizes in a simple form some of the main facts discovered in this article. It has kindly been included in [42, Section 26.2].

Theorem 5.9 For an abelian group A, the following statements are equivalent:

(1) $A$ admits a rigid ring structure.

(2) There is a group homomorphism $\phi$ such that $L_{\phi} \mathbb{Z} \cong A$.

(3) There is a map $f$ such that $L_{f} S^{1} \simeq K(A, 1)$.

Proof We first prove that (1) $\Longrightarrow(2)$. If $A$ is any rigid ring, then it follows directly from the definition (Definition 2.3) that $A$ is $\phi$-local, where $\phi: \mathbb{Z} \rightarrow A$ is the unit map. Since $\phi$ is obviously a $\phi$-equivalence, we obtain that $A \cong L_{\phi} \mathbb{Z}$. The implication (2) $\Longrightarrow$ (3) is a consequence of Theorem 5.7, and the implication (3) $\Longrightarrow$ (1) has been proved in Theorem 2.4 .

Theorem 5.7 has the following consequence. Recall that $P_{W}$ denotes localization with respect to $W \rightarrow *$.

Corollary 5.10 Let $W$ be any space.

(1) If $H^{1}(W)=0$, then $P_{W} S^{1} \simeq S^{1}$.

(2) If $H^{1}(W) \neq 0$, then $P_{W} X \simeq *$ for every connected space $X$.

Proof First, if $H^{1}(W)=0$, then $\operatorname{Hom}\left(\pi_{1}(W), \mathbb{Z}\right)$ is trivial. This implies that $\pi_{0}\left(\operatorname{map}_{*}\left(W, S^{1}\right)\right)$ is trivial, and therefore $\operatorname{map}_{*}\left(W, S^{1}\right)$ is weakly contractible, ie $S^{1}$ is $W$-null. On the other hand, if $H^{1}(W) \neq 0$, then it follows from Theorem 5.7 that $P_{W} S^{1} \simeq *$. This means that the map $S^{1} \rightarrow *$ is a $P_{W}$-equivalence, and hence all $P_{S^{1}}$-equivalences are $P_{W}$-equivalences. Therefore, $P_{W} X \simeq P_{W} P_{S^{1}} X \simeq *$ for all connected spaces $X$.

As noticed by a referee, part 2 can also be inferred from the fact that the condition $H^{1}(W) \neq 0$ implies that $S^{1}$ is a homotopy retract of $W$. 


\section{Transitional dimensions and heights}

This section contains a more detailed discussion of $f$-localizations of $K(G, n)$ when $G$ is a finite abelian group. We will need the following version of the Zabrodsky Lemma, which is discussed in [32, Theorem 1.H.1] and [74]. A space $X$ is called $f$-acyclic if $L_{f} X$ is contractible.

Lemma 6.1 For any fibration $F \rightarrow E \rightarrow X$ with $E$ and $X$ connected, if $F$ is $f$-acyclic, then the map $E \rightarrow X$ is an $f$-equivalence.

In particular, this implies that, for any connected space $X$, if the loop space $\Omega X$ is $f$-acyclic then so is $X$.

Lemma 6.2 Let $p$ be a prime and let $n$ and $r$ be any two positive integers. Then the following statements hold for every map $f$ :

(1) $K(\mathbb{Z} / p, n)$ is either $f$-local or $f$-acyclic.

(2) $K\left(\mathbb{Z} / p^{r}, n\right)$ is $f$-acyclic if and only if $K(\mathbb{Z} / p, n)$ is $f$-acyclic.

(3) If $K\left(\mathbb{Z} / p^{r}, n\right)$ is $f$-local, then $K\left(\mathbb{Z} / p^{j}, n\right)$ is $f$-local for each $j \leq r$.

Proof Write $L_{f} K(\mathbb{Z} / p, n) \simeq K(A, n) \times K(B, n+1)$ for some abelian groups $A$ and $B$. By Theorem 5.1, $A$ is a localization of $\mathbb{Z} / p$, and Corollary 4.5 tells us that either $A=0$ or $A \cong \mathbb{Z} / p$. If $A \cong \mathbb{Z} / p$, then $K(\mathbb{Z} / p, n)$ was already $f$-local. If $A=0$, then part 2 of Theorem 2.1 says that $\operatorname{Hom}(\mathbb{Z} / p, B)=0$, and hence $B$ is $p$-torsion free. Furthermore, part 3 of Theorem 2.1 implies that $\operatorname{Hom}(B, B) \cong \operatorname{Ext}(\mathbb{Z} / p, B) \cong B / p B$, and since $\operatorname{Hom}(B, B)$ has to be $p$-torsion free, it follows that $\operatorname{Hom}(B, B)=0$; thus $B=0$. In fact, the same conclusion follows from Corollary 2.10. Hence $L_{f} K(\mathbb{Z} / p, n)$ is contractible as claimed in (1).

To prove (2), suppose that $K(\mathbb{Z} / p, n)$ is $f$-acyclic, and apply Lemma 6.1 and induction to the fibrations

$$
K(\mathbb{Z} / p, n) \longrightarrow K\left(\mathbb{Z} / p^{j}, n\right) \longrightarrow K\left(\mathbb{Z} / p^{j-1}, n\right),
$$

where $j \leq r$, to infer that $K\left(\mathbb{Z} / p^{r}, n\right)$ is $f$-acyclic. Conversely, suppose that $K\left(\mathbb{Z} / p^{r}, n\right)$ is $f$-acyclic and $K(\mathbb{Z} / p, n)$ is not. Then by $(1), K(\mathbb{Z} / p, n)$ is $f$-local. If we apply Lemma 6.1 to the fibration

$$
K\left(\mathbb{Z} / p^{r}, n\right) \longrightarrow K(\mathbb{Z} / p, n) \longrightarrow K\left(\mathbb{Z} / p^{r-1}, n+1\right),
$$

we obtain that $L_{f} K\left(\mathbb{Z} / p^{r-1}, n+1\right) \simeq K(\mathbb{Z} / p, n)$, which is impossible. 
Finally, suppose that $K\left(\mathbb{Z} / p^{r}, n\right)$ is $f$-local. Then it follows from (2) that $K(\mathbb{Z} / p, n)$ is not $f$-acyclic, and hence it is $f$-local. In order to prove that $K\left(\mathbb{Z} / p^{j}, n\right)$ is $f$-local for each $j \leq r$, argue by downward induction using the fibrations

$$
K\left(\mathbb{Z} / p^{j-1}, n\right) \longrightarrow K\left(\mathbb{Z} / p^{j}, n\right) \longrightarrow K(\mathbb{Z} / p, n)
$$

and the fact that the homotopy fiber of any map between $f$-local spaces is $f$-local.

For each prime $p$, let $n_{p}(f)$ denote the supremum of the positive integers $n$ such that $K(\mathbb{Z} / p, n)$ is $f$-local. If no such integer exists, then we set $n_{p}(f)=0$. If all integers $n$ fulfill this condition, then we write $n_{p}(f)=\infty$. This is called the $\bmod p$ transitional dimension of $f$. Thus, for any map $f$, we have $n_{p}(f)=n$ if and only if the homomorphism $H^{i}(f ; \mathbb{Z} / p)$ is an isomorphism for $i \leq n$ but not for $i=n+1$. Likewise, $n_{p}(f)=\infty$ if and only if $f$ is a $\bmod p$ equivalence.

For a space $W$, we denote by $n_{p}(W)$ the dimension $n_{p}(f)$, where $f: W \rightarrow *$. Using the natural isomorphism $H^{j}(W ; \mathbb{Z} / p) \cong \operatorname{Hom}\left(H_{j}(W ; \mathbb{Z} / p), \mathbb{Z} / p\right)$ for all $j$, we see that, for a space $W$, the following statements are equivalent:

(1) $n_{p}(W)=n$.

(2) $\tilde{H}^{j}(W ; \mathbb{Z} / p)=0$ for $j \leq n$, and $H^{n+1}(W ; \mathbb{Z} / p) \neq 0$.

(3) $\tilde{H}_{j}(W ; \mathbb{Z} / p)=0$ for $j \leq n$, and $H_{n+1}(W ; \mathbb{Z} / p) \neq 0$.

Note that $n_{p}(\Sigma f)=n_{p}(f)+1$ for every map $f$, and for two maps $f$ and $g$, if there is a natural transformation $L_{g} \rightarrow L_{f}$ compatible with the units Id $\rightarrow L_{f}$ and Id $\rightarrow L_{g}$, then every $f$-local space is $g$-local [73, Section 3]; this implies that $n_{p}(f) \leq n_{p}(g)$.

A space $A$ is said to be a universal $f$-acyclic space if the two conditions $L_{f} X \simeq *$ and $P_{A} X \simeq *$ are equivalent for each space $X$. It was proved in [10, Theorem 4.4] that universal $f$-acyclic spaces exist for each map $f$; however, such a space $A$ is not homotopy unique with the given property in general - instead, it is determined up to nullity equivalence in the sense of [9].

Corollary 6.3 For a map $f: W \rightarrow V$, if $C$ denotes the homotopy cofiber of $f$, and $A$ is a universal $f$-acyclic space, then

$$
n_{p}(f)=n_{p}(A) \leq n_{p}(C) \leq n_{p}(f)+1 .
$$

Moreover, $n_{p}(f)=n_{p}(C)$ if and only if $H_{n+1}(C ; \mathbb{Z} / p) \neq 0$, where $n=n_{p}(f)$.

Proof This follows from the sequence of natural transformations

$$
L_{\Sigma f} \longrightarrow P_{C} \longrightarrow P_{A} \longrightarrow L_{f}
$$


together with the mod $p$ homology long exact sequence associated with the cofiber sequence $W \rightarrow V \rightarrow C$.

Lemma 6.4 For a map $f$, if $n<n_{p}(f)$, then $K\left(\mathbb{Z} / p^{r}, n\right)$ is $f$-local for every $r$.

Proof By assumption, both $K(\mathbb{Z} / p, n)$ and $K(\mathbb{Z} / p, n+1)$ are $f$-local. Hence we may argue by induction, using the fibrations

$$
K\left(\mathbb{Z} / p^{j}, n\right) \longrightarrow K\left(\mathbb{Z} / p^{j-1}, n\right) \longrightarrow K(\mathbb{Z} / p, n+1) .
$$

Now we can associate another number to each map $f$. For any prime $p$, let $i_{p}(f)$ be the supremum of all integers $i$ such that the space $K\left(\mathbb{Z} / p^{i}, n_{p}(f)\right)$ is $f$-local. If all integers $i$ fulfill this condition, then $i_{p}(f)=\infty$. Thus, if $n=n_{p}(f)$ (implying that $H^{n}(f ; \mathbb{Z} / p)$ is an isomorphism), then $i_{p}(f)=i$ if and only if $H^{n}\left(f ; \mathbb{Z} / p^{j}\right)$ is an isomorphism for $j \leq i$ but not for $j=i+1$. We call this number $i_{p}(f)$ the height of $f$ at the $\bmod p$ transitional dimension.

By Lemma 6.2, if $n>n_{p}(f)$ then $K(\mathbb{Z} / p, n)$ is $f$-acyclic, and so is $K\left(\mathbb{Z} / p^{r}, n\right)$ for every $r$. If $n<n_{p}(f)$, then it follows from Lemma 6.4 that $K\left(\mathbb{Z} / p^{r}, n\right)$ is $f$-local for all $r$. The general result, including the case $n=n_{p}(f)$, reads as follows.

Theorem 6.5 For any map $f$ and arbitrary integers $n, r \geq 1$, we have

$$
L_{f} K\left(\mathbb{Z} / p^{r}, n\right) \simeq \begin{cases}* & \text { if } n>n_{p}(f), \\ K\left(\mathbb{Z} / p^{i_{p}(f)}, n\right) & \text { if } n=n_{p}(f) \text { and } r \geq i_{p}(f), \\ K\left(\mathbb{Z} / p^{r}, n\right) & \text { otherwise. }\end{cases}
$$

Proof After our previous remarks, only the case $n=n_{p}(f)$ requires a proof. Write $L_{f} K\left(\mathbb{Z} / p^{r}, n\right) \simeq K(A, n) \times K(B, n+1)$. Corollary 2.10 tells us that $B=0$. If $A=0$, then $K(\mathbb{Z} / p, n)$ is $f$-acyclic, contradicting our choice of $n$. Thus, Theorem 5.1 implies that $A$ is a localization of $\mathbb{Z} / p^{r}$, and Theorem 4.10 tells us that $A=\mathbb{Z} / p^{j}$ for some $j \leq r$. We want to prove that $j=i_{p}(f)$. For this, suppose instead that $j<i_{p}(f)$. Then $K\left(\mathbb{Z} / p^{j+1}, n\right)$ is $f$-local. This yields an isomorphism

$$
\operatorname{Hom}\left(\mathbb{Z} / p^{j}, \mathbb{Z} / p^{j+1}\right) \cong \operatorname{Hom}\left(\mathbb{Z} / p^{r}, \mathbb{Z} / p^{j+1}\right),
$$

where the left-hand side equals $\mathbb{Z} / p^{j}$ and the right-hand side equals $\mathbb{Z} / p^{j+1}$. This contradiction completes the argument.

Moreover, when $n=n_{p}(f)$ and $r>i=i_{p}(f)$, the localization map

$$
\eta: K\left(\mathbb{Z} / p^{r}, n\right) \longrightarrow K\left(\mathbb{Z} / p^{i}, n\right)
$$

coincides, up to homotopy, with the map induced by the projection $\mathbb{Z} / p^{r} \rightarrow \mathbb{Z} / p^{i}$, by Theorems 4.10 and 5.1 . 
Example 6.6 If the map $f$ is of the form $W \rightarrow *$, then $i_{p}(f)=\infty$. Therefore, for any space $W$, we have

$$
P_{W} K\left(\mathbb{Z} / p^{r}, n\right) \simeq \begin{cases}* & \text { if } n>n_{p}(W), \\ K\left(\mathbb{Z} / p^{r}, n\right) & \text { otherwise. }\end{cases}
$$

This result was communicated to us by Chachólski and was, in fact, one of the starting points of our study.

Example 6.7 Let $f: K(\mathbb{Z}, n) \rightarrow K\left(\mathbb{Z} / p^{i}, n\right)$ be the map induced by the projection of $\mathbb{Z}$ onto $\mathbb{Z} / p^{i}$, where $n \geq 1$. Then $K(\mathbb{Z} / p, n)$ is $f$-local, but $K(\mathbb{Z} / p, n+1)$ is not. Hence $n_{p}(f)=n$. Likewise, $K\left(\mathbb{Z} / p^{i}, n\right)$ is $f$-local, but $K\left(\mathbb{Z} / p^{i+1}, n\right)$ is not, which implies that $i_{p}(f)=i$. This shows that all heights can occur in practice, and it follows from Theorem 6.5 that $L_{f} K\left(\mathbb{Z} / p^{r}, n\right) \simeq K\left(\mathbb{Z} / p^{i}, n\right)$ for $r \geq i$.

The following additional remark was pertinently suggested by a referee. The rational transitional dimension $n_{0}(f)$ of a map $f$ is defined, similarly as in the mod $p$ case, as the supremum of the positive integers $n$ such that $K(\mathbb{Q}, n)$ is $f$-local. If there is no such $n$, then $n_{0}(f)=0$.

Theorem 6.8 Let $A=\pi_{n}\left(L_{f}(K(\mathbb{Z}, n))\right.$ for a map $f$ and $n \geq 1$.

(1) If $n_{0}(f) \geq n$, then $A$ is a solid ring.

(2) If $n_{0}(f)<n-1$, then $\operatorname{Hom}(\mathbb{Q}, A)=0$ and $\operatorname{Ext}(\mathbb{Q}, A)=0$.

Proof If $n_{0}(f) \geq n$, then $K(\mathbb{Q}, n)$ is $f$-local. This implies that $\operatorname{Hom}(A, \mathbb{Q}) \cong \mathbb{Q}$, and therefore $A$ is a rigid ring whose underlying abelian group has rank 1. According to Proposition 3.7, this implies that $A$ is solid.

If $n_{0}(f)<n-1$, then neither $K(\mathbb{Q}, n)$ nor $K(\mathbb{Q}, n-1)$ is $f$-local and hence they are necessarily $f$-acyclic by Corollary 2.9, Theorem 5.1, and Corollary 4.5. This implies that $[K(\mathbb{Q}, n), K(A, n)]=0$ and $[K(\mathbb{Q}, n-1), K(A, n)]=0$, from which the claim in part 2 follows.

The rigid rings $A$ such that $\operatorname{Hom}(\mathbb{Q}, A)=0$ and $\operatorname{Ext}(\mathbb{Q}, A)=0$ (which is to say that $A$ is reduced and cotorsion) were classified in [12, Corollary 3.5], where it was shown that they are precisely those of the form $\prod_{p \in U} \mathbb{Z} / p^{k(p)} \oplus \prod_{q \in V} \hat{\mathbb{Z}}_{q}$ for disjoint sets of primes $U$ and $V$, and nonnegative integers $k(p)$. Therefore, the rigid ring $A=\pi_{n}\left(L_{f}(K(\mathbb{Z}, n))\right.$ can have arbitrarily large cardinality only in the case when $n_{0}(f)=n-1$. 


\section{Effect of localizations on higher homotopy groups}

In this last section, we explain how knowledge of $L_{f} K(\mathbb{Z}, n)$ or $L_{f} K\left(\mathbb{Z} / p^{r}, n\right)$ provides highly relevant information about the homotopy groups of $L_{f} X$ for other spaces $X$. The following result improves Theorem 2.6.

Theorem 7.1 Let $f$ be any map and let $A=\pi_{n}\left(L_{f} K(\mathbb{Z}, n)\right)$, where $n \geq 1$. Let $X$ be a GEM. For $m \geq n$, consider the group $G=\pi_{m}\left(L_{f} X\right)$. Then the following hold:

(1) $G \cong \operatorname{Hom}(A, G)$.

(2) $G$ admits a unique $A$-module structure.

(3) If $m>n$, then $\operatorname{Ext}(A, G)=0$.

Proof If $X$ is a GEM and $G=\pi_{m}\left(L_{f} X\right)$ where $m \geq 1$, then $K(G, m)$ is a homotopy retract of $L_{f} X$, and hence it is $f$-local. If $m \geq n$, then $K(G, n) \simeq \Omega^{m-n} K(G, m)$, and hence $K(G, n)$ is $f$-local, too. Hence (1) follows from the fact that the localization map $\eta: K(\mathbb{Z}, n) \rightarrow K(A, n)$ is an $f$-equivalence and therefore induces an isomorphism

$$
\operatorname{Hom}(A, G) \cong \operatorname{Hom}(\mathbb{Z}, G) .
$$

This isomorphism says precisely that $G$ is $\alpha$-local, where $\alpha: \mathbb{Z} \rightarrow A$ is induced by $\eta$. By Lemma 4.4, $\alpha \otimes G: \mathbb{Z} \otimes G \rightarrow A \otimes G$ is an $\alpha$-equivalence, and this implies that there is a unique $A$-module structure $A \otimes G \rightarrow G$, namely the one given by (7-1).

If $m>n$, then $K(G, n+1)$ is $f$-local, and this fact yields (3).

The $A$-modules $G$ that satisfy (7-1), or equivalently

$$
\operatorname{Hom}(A, G)=\operatorname{Hom}_{A}(A, G),
$$

were called $E$-modules by Pierce in [59]; see also [12, Section 2]. This notion was generalized and studied further in [72] for algebras $A$ over any commutative ring $R$.

Of course, neither (7-1) nor (7-2) impose any restriction on $G$ if $A=\mathbb{Z}$ or, more generally, if $\mathbb{Z} \rightarrow A$ is a ring epimorphism (ie if the ring $A$ is solid). Indeed, if $\mathbb{Z} \rightarrow A$ is a ring epimorphism, then (7-2) holds by [71, Proposition XI.1.2] for every $A$-module $G$. However, if $\mathbb{Z} \rightarrow A$ is not a ring epimorphism, then there is at least one ring $G$ and two distinct ring homomorphisms $A \rightarrow G$ such that the composites $\mathbb{Z} \rightarrow G$ are equal. Then $G$ becomes an $A$-module, which violates (7-1). Hence condition (7-1) imposes a nonvoid restriction on the $A$-module $G$ precisely when the ring $A$ is rigid but not solid. For example, if $A=\widehat{\mathbb{Z}}_{p}$ and $G=\widehat{\mathbb{Q}}_{p}$, then (7-1) does not hold. 
Corollary 7.2 Suppose that $L_{f} K(\mathbb{Z}, n) \simeq K(\mathbb{Z} / t, n)$, where $t$ is any positive integer and $n \geq 1$. If $X$ is a GEM, then $\pi_{m}\left(L_{f} X\right)=0$ for $m>n$.

Proof From Theorem 7.1, we know that each of the homotopy groups $\pi_{m}\left(L_{f} X\right)$ is a $\mathbb{Z} / t$-module for $m \geq n$. However, if an abelian group $G$ satisfies $t G=0$ and $\operatorname{Ext}(\mathbb{Z} / t, G)=0$, then $G=0$.

The conclusion of Corollary 7.2 seems to hold for a much broader class of spaces, not necessarily products of Eilenberg-Mac Lane spaces. Perhaps the answer to the following question is affirmative. Many unsuccessful attempts have been made to find an answer, so it seems to be a difficult problem.

Question 7.3 Let $t$ be any positive integer. If $f: S^{1} \rightarrow K(\mathbb{Z} / t, 1)$ is a map inducing the projection $\mathbb{Z} \rightarrow \mathbb{Z} / t$ on fundamental groups, is it true that $\pi_{m}\left(L_{f} X\right)=0$ for all spaces $X$ and $m \geq 2$ ?

Observe that, if $A$ is a rigid ring and the unit map $\mathbb{Z} \rightarrow A$ is not injective, then $A \cong \mathbb{Z} / t$ for some integer $t$. Indeed, if the identity element of $A$ has finite order, then $t A=0$ for some integer $t$, and for a rigid ring, this implies that $A$ is cyclic; this fact was already noted in [70]. Therefore, if $L_{f} K(\mathbb{Z}, n) \simeq K(A, n)$, then either $A$ is cyclic or the induced map $\mathbb{Z} \rightarrow A$ is a proper monomorphism. We next address the latter case.

We recall from [5] and [8, Lemma 5.5] that, given an abelian group $B$, if $P$ denotes the set of primes $p$ for which the map $x \mapsto p x$ is an automorphism of $B$, then the class of abelian groups $G$ such that $\operatorname{Hom}(B, G)=0$ and $\operatorname{Ext}(B, G)=0$ consists precisely of the $P$-local groups if $B$ is torsion, and it consists of the Ext- $P$-complete abelian groups (2-8) otherwise.

Theorem 7.4 Suppose that $L_{f} K(\mathbb{Z}, n) \simeq K(A, n)$, where $n \geq 1$ and $A$ is not cyclic. Let $X$ be any GEM, and let $P$ be the set of primes $p$ such that multiplication by $p$ is an automorphism of $A / \mathbb{Z}$. If $A / \mathbb{Z}$ is torsion, then $\pi_{m}\left(L_{f} X\right)$ is $P$-local if $m>n$. If $A / \mathbb{Z}$ has elements of infinite order, then $\pi_{m}\left(L_{f} X\right)$ is Ext- $P$-complete if $m>n$.

Proof Let $G=\pi_{m}\left(L_{f} X\right)$ with $m>n$. By Theorem 7.1, we have $\operatorname{Ext}(A, G)=0$ and $\operatorname{Hom}(A, G) \cong \operatorname{Hom}(\mathbb{Z}, G)$. Hence, by applying the functor $\operatorname{Hom}(-, G)$ to the short exact sequence $0 \rightarrow \mathbb{Z} \rightarrow A \rightarrow A / \mathbb{Z} \rightarrow 0$, we infer that

$$
\operatorname{Hom}(A / \mathbb{Z}, G)=0=\operatorname{Ext}(A / \mathbb{Z}, G),
$$

so that our claim follows from [8, Lemma 5.5]. 
Theorem 7.4 is conveniently illustrated by ordinary homological localization with coefficients in $\mathbb{Z}_{(p)}$ or $\mathbb{Z} / p$, and even better by localization with respect to Morava $K$-theories; see [7, Examples 7.4 and 7.5].

Theorem 7.5 Let $f$ be any map and $p$ a prime. Suppose that the transitional dimension $n_{p}(f)$ is finite. If $X$ is any GEM, then:

(1) The group $\pi_{m}\left(L_{f} X\right)$ is a $\mathbb{Z}[1 / p]$-module if $m \geq n_{p}(f)+2$, and $\pi_{m}\left(L_{f} X\right)$ is $p$-torsion free if $m=n_{p}(f)+1$.

(2) Suppose that the height $i_{p}(f)$ is finite. Then $\pi_{m}\left(L_{f} X\right)$ is a $\mathbb{Z}[1 / p]$-module if $m \geq n_{p}(f)+1$, and the $p$-torsion subgroup of $\pi_{m}\left(L_{f} X\right)$ is annihilated by $p^{i_{p}(f)}$ if $m=n_{p}(f)$.

Proof If $m \geq n_{p}(f)+1$ and we write $G=\pi_{m}\left(L_{f} X\right)$, then $K(\mathbb{Z} / p, m)$ is $f$-acyclic and $K(G, m)$ is $f$-local. It follows that $\operatorname{Hom}(\mathbb{Z} / p, G)=0$, and hence $G$ is $p$-torsion free. If $m \geq n_{p}(f)+2$, then we also have $\operatorname{Ext}(\mathbb{Z} / p, G)=0$, which, together with the fact that $G$ is $p$-torsion free, guarantees that $G$ is a $\mathbb{Z}[1 / p]$-module.

If $i=i_{p}(f)$ is finite, then it follows from Theorem 6.5 that the natural map

$$
K\left(\mathbb{Z} / p^{r+1}, n_{p}(f)\right) \longrightarrow K\left(\mathbb{Z} / p^{r}, n_{p}(f)\right)
$$

is an $f$-equivalence for $r \geq i$. If $m=n_{p}(f)+1$, then

$$
\operatorname{Ext}\left(\mathbb{Z} / p^{r}, G\right) \cong \operatorname{Ext}\left(\mathbb{Z} / p^{r+1}, G\right)
$$

for $r \geq i$. Hence $\operatorname{Ext}(\mathbb{Z} / p, G)=0$, and we infer again that $G$ is a $\mathbb{Z}[1 / p]$-module. Finally, if $m=n_{p}(f)$, then we deduce that $\operatorname{Hom}\left(\mathbb{Z} / p^{i}, G\right) \cong \operatorname{Hom}\left(\mathbb{Z} / p^{r}, G\right)$ for $r \geq i$, from which it follows that the $p$-torsion subgroup of $G$ is a $\mathbb{Z} / p^{i}$-module.

Example 7.6 For the map $f: K(\mathbb{Z} / p, 1) \rightarrow *$, we have $n_{p}(f)=0$, which implies, by Theorem 7.4 , that the homotopy groups of any $f$-local GEM are $\mathbb{Z}[1 / p]$-modules in dimensions higher than 1 . Indeed, it follows from the fibration

$$
K\left(\mathbb{Z} / p^{\infty}, n-1\right) \longrightarrow K(\mathbb{Z}, n) \longrightarrow K(\mathbb{Z}[1 / p], n)
$$

that $L_{f} K(\mathbb{Z}, n) \simeq K(\mathbb{Z}[1 / p], n)$ for $n \geq 2$; see [17, Section 7]. A similar argument shows that $L_{f} K(G, n) \simeq K(G \otimes \mathbb{Z}[1 / p], n)$ for every abelian group $G$ and each $n \geq 2$. On the other hand, all finite-dimensional CW-complexes are $f$-local by Miller's main theorem in [53], yet their homotopy groups need not be $\mathbb{Z}[1 / p]$-modules. This shows that the above theorems are false if we omit the assumption that $X$ is a GEM. 
Example 7.7 Let $f$ be any map such that $L_{f}$ is localization with respect to complex $K$-homology. Since $K(\mathbb{Z} / p, 1)$ is $K$-local and $K(\mathbb{Z} / p, 2)$ is $K$-acyclic for all primes $p$ (see [7] or [54]), it follows that $n_{p}(f)=1$ for every $p$. Thus Theorem 7.5 tells us that if $X$ is any GEM, then the homotopy groups $\pi_{m}\left(X_{K}\right)$ of the $K$-localization of $X$ are $\mathbb{Q}$-vector spaces if $m \geq 3$, and $\pi_{2}\left(X_{K}\right)$ is torsion-free. This observation sheds new light on [54, Theorem 3.2]. Indeed, if $X$ is any 2-connected GEM, then $X_{K}$ is a 2 -connected rational GEM. Since the class of $K$-equivalences with rational coefficients is equal to the class of ordinary rational homology equivalences [54, Lemma 1.8], the rationalization $X_{0}$ is $K$-local. From this fact, it follows directly that $X_{K} \simeq X_{0}$ if $X$ is any $2-$ connected GEM.

\section{References}

[1] J Adámek, J Rosický, Locally presentable and accessible categories, London Mathematical Society Lecture Note Series 189, Cambridge Univ. Press (1994) MR

[2] B Badzioch, Recognition principle for generalized Eilenberg-Mac Lane spaces, from: "Cohomological methods in homotopy theory", (J Aguadé, C Broto, C Casacuberta, editors), Progr. Math. 196, Birkhäuser, Basel (2001) 21-26 MR

[3] B Badzioch, M Feshbach, A note on localizations of perfect groups, Proc. Amer. Math. Soc. 133 (2005) 693-697 MR

[4] M Blomgren, W Chachólski, E D Farjoun, Y Segev, Idempotent transformations of finite groups, Adv. Math. 233 (2013) 56-86 MR

[5] A K Bousfield, Types of acyclicity, J. Pure Appl. Algebra 4 (1974) 293-298 MR

[6] A K Bousfield, Constructions of factorization systems in categories, J. Pure Appl. Algebra 9 (1976/77) 207-220 MR

[7] A K Bousfield, On homology equivalences and homological localizations of spaces, Amer. J. Math. 104 (1982) 1025-1042 MR

[8] A K Bousfield, Localization and periodicity in unstable homotopy theory, J. Amer. Math. Soc. 7 (1994) 831-873 MR

[9] A K Bousfield, Unstable localization and periodicity, from: "Algebraic topology: new trends in localization and periodicity", (C Broto, C Casacuberta, G Mislin, editors), Progr. Math. 136, Birkhäuser, Basel (1996) 33-50 MR

[10] A K Bousfield, Homotopical localizations of spaces, Amer. J. Math. 119 (1997) 13211354 MR

[11] A K Bousfield, D M Kan, The core of a ring, J. Pure Appl. Algebra 2 (1972) 73-81 MR

[12] R A Bowshell, P Schultz, Unital rings whose additive endomorphisms commute, Math. Ann. 228 (1977) 197-214 MR 
[13] G Braun, R Göbel, E-algebras whose torsion part is not cyclic, Proc. Amer. Math. Soc. 133 (2005) 2251-2258 MR

[14] K S Brown, Cohomology of groups, Graduate Texts in Mathematics 87, Springer, New York (1982) MR

[15] J Buckner, M Dugas, Co-local subgroups of abelian groups, from: "Abelian groups, rings, modules, and homological algebra", (P Goeters, O M G Jenda, editors), Lect. Notes Pure Appl. Math. 249, Chapman \& Hall/CRC, Boca Raton, FL (2006) 29-37 MR

[16] J Buckner, M Dugas, Co-local subgroups of nilpotent groups of class 2, from: "Models, modules and abelian groups", (R Göbel, B Goldsmith, editors), Walter de Gruyter, Berlin (2008) 351-358 MR

[17] C Casacuberta, Recent advances in unstable localization, from: "The Hilton Symposium 1993”, (G Mislin, editor), CRM Proc. Lecture Notes 6, Amer. Math. Soc. (1994) 1-22 MR

[18] C Casacuberta, Anderson localization from a modern point of view, from: “The Čech centennial”, (M Cenk1, H Miller, editors), Contemp. Math. 181, Amer. Math. Soc. (1995) 35-44 MR

[19] C Casacuberta, On structures preserved by idempotent transformations of groups and homotopy types, from: "Crystallographic groups and their generalizations", (P Igodt, H Abels, Y Félix, F Grunewald, editors), Contemp. Math. 262, Amer. Math. Soc. (2000) 39-68 MR

[20] C Casacuberta, J J Gutiérrez, Homotopical localizations of module spectra, Trans. Amer. Math. Soc. 357 (2005) 2753-2770 MR

[21] C Casacuberta, J J Gutiérrez, I Moerdijk, R M Vogt, Localization of algebras over coloured operads, Proc. Lond. Math. Soc. 101 (2010) 105-136 MR

[22] C Casacuberta, O Raventós, A Tonks, Comparing localizations across adjunctions, preprint (2014) arXiv

[23] C Casacuberta, J L Rodríguez, On towers approximating homological localizations, J. London Math. Soc. 56 (1997) 645-656 MR

[24] W Chachólski, E Damian, E D Farjoun, Y Segev, The A-core and A-cover of a group, J. Algebra 321 (2009) 631-666 MR

[25] W Dicks, W Stephenson, Epimorphs and dominions of Dedekind domains, J. London Math. Soc. 29 (1984) 224-228 MR

[26] A Dold, R Thom, Quasifaserungen und unendliche symmetrische Produkte, Ann. of Math. 67 (1958) 239-281 MR

[27] M Dugas, Localizations of torsion-free abelian groups, J. Algebra 278 (2004) 411-429 MR 
[28] M Dugas, Localizations of torsion-free abelian groups, II, J. Algebra 284 (2005) 811-823 MR

[29] M Dugas, Co-local subgroups of abelian groups, II, J. Pure Appl. Algebra 208 (2007) 117-126 MR

[30] M Dugas, A Mader, C Vinsonhaler, Large E-rings exist, J. Algebra 108 (1987) 88-101 MR

[31] W G Dwyer, J H Palmieri, Ohkawa's theorem: there is a set of Bousfield classes, Proc. Amer. Math. Soc. 129 (2001) 881-886 MR

[32] E D Farjoun, Cellular spaces, null spaces and homotopy localization, Lecture Notes in Mathematics 1622, Springer (1996) MR

[33] E D Farjoun, R Göbel, Y Segev, Cellular covers of groups, J. Pure Appl. Algebra 208 (2007) 61-76 MR

[34] E D Farjoun, R Göbel, Y Segev, S Shelah, On kernels of cellular covers, Groups Geom. Dyn. 1 (2007) 409-419 MR

[35] L Fuchs, R Göbel, Cellular covers of abelian groups, Results Math. 53 (2009) 59-76 MR

[36] R Göbel, B Goldsmith, Classifying E-algebras over Dedekind domains, J. Algebra 306 (2006) 566-575 MR

[37] R Göbel, J L Rodríguez, S Shelah, Large localizations of finite simple groups, J. Reine Angew. Math. 550 (2002) 1-24 MR

[38] R Göbel, J L Rodríguez, L Strüngmann, Cellular covers of cotorsion-free modules, Fund. Math. 217 (2012) 211-231 MR

[39] R Göbel, S Shelah, Constructing simple groups for localizations, Comm. Algebra 30 (2002) 809-837 MR

[40] R Göbel, L Strüngmann, Almost-free $E(R)$-algebras and $E(A, R)$-modules, Fund. Math. 169 (2001) 175-192 MR

[41] R Göbel, J Trlifaj, Approximations and endomorphism algebras of modules, de Gruyter Expositions in Mathematics 41, Walter de Gruyter, Berlin (2006) MR

[42] R Göbel, J Trlifaj, Approximations and endomorphism algebras of modules, 2: Predictions, extended edition, de Gruyter Expositions in Mathematics 41, Walter de Gruyter, Berlin (2012) MR

[43] J J Gutiérrez, Localización y conservación de estructuras en homotopía estable, PhD thesis, Universitat de Barcelona (2004) Available at http://diposit.ub.edu/ dspace/handle/2445/35147

[44] J J Gutiérrez, Homological localizations of Eilenberg-MacLane spectra, Forum Math. 22 (2010) 349-356 MR 
[45] J J Gutiérrez, Cellularization of structures in stable homotopy categories, Math. Proc. Cambridge Philos. Soc. 153 (2012) 399-418 MR

[46] A Hatcher, Algebraic topology, Cambridge Univ. Press (2002) MR

[47] PS Hirschhorn, Model categories and their localizations, Mathematical Surveys and Monographs 99, Amer. Math. Soc. (2003) MR

[48] I Kaplansky, Infinite abelian groups, 2nd edition, The University of Michigan Press (1969) MR

[49] A Libman, Cardinality and nilpotency of localizations of groups and G-modules, Israel J. Math. 117 (2000) 221-237 MR

[50] S Mac Lane, Categories for the working mathematician, Graduate Texts in Mathematics 5, Springer, New York (1971) MR

[51] J P May, Simplicial objects in algebraic topology, Van Nostrand Mathematical Studies 11, D Van Nostrand Co, Princeton (1967) MR

[52] J P May, Infinite loop space theory, Bull. Amer. Math. Soc. 83 (1977) 456-494 MR

[53] H Miller, The Sullivan conjecture on maps from classifying spaces, Ann. of Math. 120 (1984) 39-87 MR

[54] G Mislin, Localization with respect to K-theory, J. Pure Appl. Algebra 10 (1977/78) 201-213 MR

[55] R J Nunke, Modules of extensions over Dedekind rings, Illinois J. Math. 3 (1959) 222-241 MR

[56] T Ohkawa, The injective hull of homotopy types with respect to generalized homology functors, Hiroshima Math. J. 19 (1989) 631-639 MR

[57] N O'Sullivan, Localizations of free soluble groups, J. Group Theory 4 (2001) 89-96 MR

[58] C Parker, J Saxl, Localization and finite simple groups, Israel J. Math. 153 (2006) 285-305 MR

[59] R S Pierce, E-modules, from: "Abelian group theory", (L Fuchs, R Göbel, P Schultz, editors), Contemp. Math. 87, Amer. Math. Soc. (1989) 221-240 MR

[60] R S Pierce, C I Vinsonhaler, Classifying E-rings, Comm. Algebra 19 (1991) 615-653 MR

[61] D J S Robinson, A course in the theory of groups, 2nd edition, Graduate Texts in Mathematics 80, Springer, New York (1996) MR

[62] J L Rodríguez, On homotopy colimits of spaces with a single homology or homotopy group, PhD thesis, Universitat Autònoma de Barcelona (1997)

[63] J L Rodríguez, J Scherer, Cellular approximations using Moore spaces, from: “Cohomological methods in homotopy theory", (J Aguadé, C Broto, C Casacuberta, editors), Progr. Math. 196, Birkhäuser, Basel (2001) 357-374 MR 
[64] J L Rodríguez, J Scherer, A connection between cellularization for groups and spaces via two-complexes, J. Pure Appl. Algebra 212 (2008) 1664-1673 MR

[65] J L Rodríguez, J Scherer, L Strüngmann, On localization of torsion abelian groups, Fund. Math. 183 (2004) 123-138 MR

[66] J L Rodríguez, J Scherer, J Thévenaz, Finite simple groups and localization, Israel J. Math. 131 (2002) 185-202 MR

[67] J L Rodríguez, J Scherer, A Viruel, Preservation of perfectness and acyclicity: Berrick and Casacuberta's universal acyclic space localized at a set of primes, Forum Math. 17 (2005) 67-75 MR

[68] J L Rodríguez, J Scherer, A Viruel, Non-simple localizations of finite simple groups, J. Algebra 305 (2006) 765-774 MR

[69] J L Rodríguez, L Strüngmann, On cellular covers with free kernels, Mediterr. J. Math. 9 (2012) 295-304 MR

[70] P Schultz, The endomorphism ring of the additive group of a ring, J. Austral. Math. Soc. 15 (1973) 60-69 MR

[71] B Stenström, Rings of quotients, Grundl. Math. Wissen. 217, Springer, New York (1975) MR

[72] L Strüngmann, Almost-free $E(R)$-algebras over countable domains, $\mathrm{PhD}$ thesis, Universität Essen (1998)

[73] J-y Tai, On $f$-localization functors and connectivity, from: "Stable and unstable homotopy”, (W G Dwyer, S Halperin, R Kane, S O Kochman, M E Mahowald, P S Selick, editors), Fields Inst. Commun. 19, Amer. Math. Soc. (1998) 285-298 MR

[74] J-y Tai, A version of Zabrodsky's lemma, Proc. Amer. Math. Soc. 126 (1998) 1573-1578 MR

[75] G W Whitehead, Elements of homotopy theory, Graduate Texts in Mathematics 61, Springer, New York (1978) MR

[76] G V Wilson, Additive groups of T-rings, Proc. Amer. Math. Soc. 99 (1987) 219-220 MR

Institut de Matemàtica, Universitat de Barcelona

Gran Via de les Corts Catalanes, 585, 08007 Barcelona, Spain

Departamento de Matemáticas, Universidad de Almería

04120 Almería, Spain

Department of Mathematics, Dartmouth College

Hanover, NH 03755-3551, United States

carles.casacuberta@ub.edu, jlrodri@ual.es,

patrick.tai@alumni.cuhk.net

Received: 30 September 2015 